\title{
M-PERT: manual project-duration estimation technique for teaching scheduling basics
}

Article

Accepted Version

Ballesteros-Pérez, P. (2017) M-PERT: manual project-duration estimation technique for teaching scheduling basics. Journal of Construction Engineering and Management, 143 (9). 04017063. ISSN 0733-9364 doi: https://doi.org/10.1061/ (ASCE)CO.1943-7862.0001358 Available at https://centaur.reading.ac.uk/69858/

It is advisable to refer to the publisher's version if you intend to cite from the work. See Guidance on citing.

Published version at: http://ascelibrary.org/doi/10.1061/\%28ASCE\%29CO. 1943-7862.0001358

To link to this article DOI: http://dx.doi.org/10.1061/(ASCE)CO.1943-

7862.0001358

Publisher: American Society of Civil Engineers

All outputs in CentAUR are protected by Intellectual Property Rights law, including copyright law. Copyright and IPR is retained by the creators or other copyright holders. Terms and conditions for use of this material are defined in the End User Agreement.

www.reading.ac.uk/centaur 


\section{CentAUR}

Central Archive at the University of Reading

Reading's research outputs online 
THANKS FOR DOWNLOADING THIS PAPER.

This is a post-refereeing version of a manuscript published by the ASCE.

Please, in order to cite this paper properly:

\author{
Ballesteros-Pérez, P. (2017) “M-PERT. A manual project duration estimation \\ technique for teaching scheduling basics." Journal of Construction Engineering and \\ Management. In press. \\ http://dx.doi.org/10.1061/(ASCE)CO.1943-7862.0001358
}

The authors recommend going to the publisher's website in order to access the full paper.

If this paper helped you somehow in your research, feel free to cite it. 


\section{M-PERT. A manual project duration estimation technique}

\section{for teaching scheduling basics}

Pablo Ballesteros-Pérez, Ph.D.

Lecturer. School of Construction Management and Engineering. University of Reading, Whiteknights, Reading RG66AW, United Kingdom.E-mail: p.ballesteros@reading.ac.uk

\section{ABSTRACT}

The Program Evaluation and Review Technique (PERT) has become a classic Project

Management tool for estimating project duration when the activities have uncertain durations.

However, despite its simplicity and widespread adoption, the original PERT, in neglecting

the merge event bias, significantly underestimated the duration average and overestimated the duration variance of real-life projects. To avoid these and other shortcomings, many authors have worked over the last 60 years at producing interesting alternative PERT extensions. This paper proposes joining the most relevant of those to create a new reformulated PERT, named M-PERT.

M-PERT is quite accurate when estimating real project duration, while also allowing for a number of interesting network modelling features the original PERT lacked: probabilistic alternative paths, activity self-loops, minima of activity sets and correlation between activities. However, unlike similar scheduling methods, M-PERT allows manual calculation through a recursive merging procedure that downsizes the network until the last standing activity represents the whole (or remaining) project duration. Hence, M-PERT constitutes an attractive tool for teaching scheduling basics to engineering students in a more intuitive way, with or without the assistance of computer-based simulations or software. One full case study will also be proposed and future research paths suggested.

\section{KEYWORDS}

Scheduling, PERT; GERT; Stochastic Network Analysis; Project duration. 
Introduction

In 1959, an Operations Research team formed by D.G. Malcom, J.H. Roseboom and C.E.

Clark from the company Booz, Allen and Hamilton in Chicago, along with W. Fazar, from the US Navy Special Projects Office in Washington, published a technique for measuring and controlling the progress of the Polaris Fleet Ballistic Missile (FBM) program (Malcolm et al. 1959). This technique was initially named the Program Evaluation Research Task and later renamed the Program Evaluation and Review Technique (PERT). It has become one of the most popular Project Management (PM) scheduling tools since then. management methodology that allowed him/her to evaluate '(a) [the] progress to date and [the] outlook for accomplishing the objectives of the FBM program, (b) [the] validity of established plans and schedules for accomplishing the program objectives, and (c) [the] effects of changes proposed in established plans' (Malcolm et al., 1959, p.646). These three aims were quite ambitious indeed, and despite one imagining PERT doing all of these with a leap of faith, what PERT actually and basically does is to estimate the probabilistic duration of any project or the remaining part of a project when the activities have uncertain (not deterministic) durations. Also, PERT can be implemented for cost estimation purposes with some technical variations (e.g. (Asmar et al. 2011)); however, for the sake of clarity we will just deal with the time dimension in this paper.

In its favor, this technique is very easy to implement and was the first of its kind for dealing with projects whose activity durations are modeled by probability distributions, or Stochastic Network Analysis (SNA) as it known as nowadays. Against it, the procedure proposed by PERT underestimates the project duration when there are multiple parallel paths, which unfortunately is the norm in construction projects. Indeed, the authors were aware of

50 this shortcoming since they very briefly mentioned in the original paper that 'this 
simplification gives biased estimates such that the estimated expected time of events are always too small' (Malcolm et al. 1959). Two publications by C.E. Clark indeed followed the publication of PERT (Clark 1961, 1962), but the solution proposed was not easy to implement, so it opened interesting avenues of research for the scientific community that have continued to this day, mostly in the Operations Research discipline.

Since the inception of PERT almost 60 years ago, the number of fields in which this technique has been applied has been quite varied. It is not only applied to military, research \& development and civil engineering projects as was originally intended (Stauber et al. 1959), but also to fields as diverse as medicine (e.g. Woolf et al. (1968)), exports (e.g. Tatterson (1974)), contracting (e.g. Mummolo (1997)) and rural development (e.g. Tavares (2002)), to cite just a few. In fact, its reach and application are virtually unlimited since it is useful for any project duration estimation purposes where activities have non-deterministic durations. However, another collateral problem has been that despite PERT being a celebrated PM technique, it also probably is one of the most widely misunderstood. In particular, many engineering and scheduling students, as well as professionals, still think that PERT is either a type of network representation, as one of the first versions of the Project Management Body of Knowledge (PMBoK) pointed out explicitly (PMI 1996), or that it is just a three-point time and/or cost estimation technique (PMI 2008). The latter misrepresentation is the consequence of the second half of the PERT method, which deals with calculating the expected project duration and its variance by means of critical path project activities, being left out of this publication.

The purpose of this paper is to update PERT and propose a newly redefined technique named M-PERT, which deals with the most relevant shortcoming of the original technique (the merge event bias), allows manual calculation, and adds a series of new interesting features that the original technique lacked. M-PERT will allow the modelling of real-life 
projects far more representatively and help scheduling students to understand more intuitively basic concepts of scheduling when activities have uncertain durations.

The number of PERT-related publications might be counted in the thousands nowadays, so we cannot reference them all. Instead, as in any other mature fields, we will undertake a systematic review of the most relevant sources that have shaped this new technique. This systematic review will be presented in the Background section under three thematic subsections. Then, an outline of the model will be presented followed by an application in the M-PERT outline and Case study sections. A Results section will gather the application outcomes while comparing the accuracy improvements achieved, whereas a final and brief Discussions and conclusions section will summarize the most important aspects and possible research directions for the model proposed.

\section{Background}

\section{$\underline{\text { An overview of PERT and its limitations }}$}

In a nutshell, PERT is a scheduling tool that is applied in two stages. First, after identifying all the activities involved in a project and stating their precedence relationships, the first stage comprises modelling their probable durations. For that purpose, the original PERT authors proposed a three-point estimation procedure. The user (or another expert on hand) is asked to state the Optimistic $(O)$, most Likely $(L)$ and Pessimistic $(P)$ durations that each activity is foreseen to have before those activities take place. Then, the mean duration $(\mu)$ and the standard deviation $(\sigma)$ from each activity are calculated by using these three duration estimates $(O, L$ and $P)$ by means of these straightforward expressions:

$$
\begin{aligned}
& =(O+4 L+P) / 6 \\
& =\left(\begin{array}{ll}
P & O
\end{array}\right) / 6
\end{aligned}
$$


100 With the values of $\mu$ and $\sigma$ for each activity, a Beta distribution which varies between

$101[O, P]$ is fitted for modelling the activity duration. However, despite the highly controversial

102 and still ongoing debate about the accuracy of both the three-point estimate procedure and the

103 choice of the Beta distribution to model the activity durations, the PERT method is only

104 interested in the $\mu$ and $\sigma$ values of each activity for the second stage of the application. This

105 second stage of PERT (not mentioned by any Project Management standard) comprises: (a)

106 identifying the activities that are in the critical path, and (b) assuming that the Project

107 duration mean $\left(\mu_{p}\right)$ and the standard deviation $\left(\sigma_{p}\right)$ are equal:

$$
\begin{gathered}
{ }_{p}=\underset{i \text { critical path }}{i} \\
p_{p}=\sqrt{i_{i \text { critical path }}^{i}}
\end{gathered}
$$

110 Now, if the project scheduler wants to know what the probabilities of ending a project in

$111 X$ days are, he/she just needs to know that the project total duration is supposed to be

112 following a Normal distribution with mean $\mu_{p}$ and standard deviation $\sigma_{p}$. Then, the specific

113 probability of $X$ happening can be calculated by means of either the mathematical expressions

114 of the cumulative Normal distribution, by resorting to a spreadsheet or, when the calculations

115 are made by hand, by standardizing the project duration as in Equation 5 and then looking up

116 the cumulative probability value of $z$ in a standard Normal table.

$$
z=\left(\begin{array}{ll}
X & p
\end{array}\right) / p
$$

118 This is the essence of the PERT method. The logical lack of trust displayed by the

119 research community since PERT was published has manifested itself as a determination to 120 see what kind of errors are involved by implementing it. In this regard, MacCrimmon and 121 Ryavec (1964) broke down PERT into every possible piece and developed the most thorough 122 analytical review that anyone has made of PERT since its publication. This piece of research 
was actually outsourced by the US Air Force to the Rand Corporation due to the consistent and systematic errors that PERT was evidencing in their project forecasts, and they divided their memorandum into two main sections. In the first section, they studied the activity duration-level errors, that is, '(1) the assumption of the Beta distribution, (2) imprecise time estimates, and (3) the assumption of the standard deviation (one-sixth of the range) and the approximation formula for calculating the mean time' (MacCrimmon and Ryavec 1964). The second section checked the accuracy of the project duration mean, variance and the (Normal) probability statements.

The results summary from the first section was that using the three-point estimates could cause a range estimation error of between $10 \%$ and $20 \%$ of the range $[O, P]$, but, despite the error of estimation of the mean $(\mu)$ and standard deviation $(\sigma)$ being likely to be bigger (from $15 \%$ to $30 \%$ ), a degree of cancellation was expected to occur depending on the network. Indeed, there is generally a high degree of cancellation as will be proven later. But the other noteworthy statement that the authors made at that time was that, to a certain extent, it was pointless having a discussion about what the right kind of distribution is - Beta or another -

138 for modelling an activity duration. Indeed, they reinforced this idea by saying that if PERT had used triangular distributions instead of Beta distributions, their analysis would have given almost identical results (MacCrimmon and Ryavec 1964). A recent study only focusing on determining the importance of the specific distribution selection in PERT also supported this

142 claim and stated that the maximum deviation by using alternative distributions in the project 143 duration an estimation is generally well below 10\% (Hajdu and Bokor 2014).

144 Nevertheless, the results from the second section of the PERT model analysis evidenced the real problem. They confirmed that unless there is one clear dominant critical path (a chain of activities whose total duration is significantly longer than the second longest path), the

147 PERT-calculated mean (equation 3) will be biased optimistically (underestimating the real 
148 project duration), while the PERT-calculated variance (equation 4) will be biased in the other

149 direction (overestimating the actual duration variance). At the time this study was published,

150 Extreme Value theory had not been properly developed, but the authors clearly identified that

151 the higher the number of parallel paths, the more positively skewed the distribution modelling

152 the project duration would become, making the Normally-distributed assumption of the

153 'total' project duration of questionable validity with multiple parallel paths.

What matters and what does not matter in PERT

The first part of the systematic review by MacCrimmon and Ryavec (1964) focused on the activity level duration modelling. They stated that the particular choice of the distribution was hardly relevant. Instead, it was the distribution (duration) mean and variance that really matters. This was an important outcome that, apparently, a big part of the research community had tried to ignore due to the extraordinary amount of research focused on improving the particular statistical distribution for better modelling of the activity duration.

In a similar vein, a recent study by Herrerías-Velasco et al. (2011), seeking to close the unending debate about whether the approximations originally proposed by the PERT authors to estimate whether the activity duration mean and variance were accurate enough, concluded that, in general, the expression of the mean (Equation 1) is very accurate, whereas the expression for the variance (Equation 2) requires to be multiplied by a correction factor $K$ whose expression is:

$$
K=\sqrt{\frac{5}{7}+\frac{16}{7} \frac{(L O)(P \quad L)}{(P \quad O)^{2}}}
$$
activity duration, Figure 1 is divided into two vertical blocks. The top half shows how the 
171 statistical distribution resulting from one, two and three activities in series evolves when the 172 activity duration is modeled by a Uniform distribution (first row) or a Beta (PERT-like)

173 distribution (second row). The case with just one activity (first column) illustrates indeed the 174 original distribution, but when more independent and identically distributed (iid) activities are 175 added in series, the resulting distribution quickly changes its shape to resemble a Normal 176 distribution. In fact, if we observed the result for five activities in series, it would be quite 177 difficult to tell them apart from a Normal distribution. Therefore, the particular statistical 178 distribution chosen hardly matters since it is difficult to find more extreme examples than a 179 Uniform and a Beta distribution, both equally and quickly converging to a Normal 180 distribution.

However, what is relevant is the original activity duration mean and standard deviation.

183 Both the Uniform and the Beta distribution had the same duration mean of $\mu=5$ (time units)

184 and it is easy to see that as we add more activities in series, the resulting mean is $n \cdot \mu$, where $n$ is the number of activities in the series ( $n \cdot \mu=10$ for two activities, 15 for three activities). On the other hand, the Uniform distribution above has a higher variance (higher standard 187 deviation) compared to the Beta distribution below which, on top of that, is positively skewed 188 and has a range of variation from 0 to 15 . It is again evident that as we add more activities in series, the resulting distribution of the Uniform is sparser (since it originally had a bigger variance), whereas the result from the Beta-distributed activities has almost removed the skewness (degree of asymmetry) while it is narrower (duration values are more concentrated 192 near the mean).

Therefore, in any project that has three or more activities in series (basically any real project), the selection of the specific statistical distribution is not relevant. What matters is to 
specify as accurately as possible the activity mean and standard deviation. The original PERT technique proposed doing this by a three-point estimate, but this caused an error of between 15 and $35 \%$, particularly concentrated in the variance estimation (when departing from the $O$, $L$ and $P$ estimates). The obvious solution is to get rid of the intermediate step and ask the

199 scheduler (or the field expert) to directly provide a mean and a standard deviation for each activity duration, and if he/she feels unsure about quantifying particularly the variance, then resort to the original PERT expressions (equations 1 and 2) with the correction factor stated in Equation 6.

With the activity-level duration issues clarified, it is time to address another PERT shortcoming: the result of having multiple parallel paths in a network. To exemplify this, we will make use of the bottom half of Figure 1. But first, let us propose an example.

Imagine that the activity duration can be modeled with a fair six-sided die. Then, the activity duration might be $\{1,2,3,4,5,6\}$, with equal probability each of $1 / 6$ (a discrete Uniform distribution). The average of these six possible outcomes would be 3.5 (time units), So, if we cast two dice, the average duration would be two times 3.5 , that is, 7 . If we cast 210 three dice the average duration would be three times 3.5, that is, 10.5 . This is the effect of 211 adding activities in series; we are just 'adding' distributions (convolution of distributions), as in the upper half of Figure 1. But when there are several parallel paths, we are not doing sums anymore: we are taking the 'maxima' (computing the Cumulative Distribution Function of a maximum) of the different path durations. In our dice example, imagine that we have two

215 parallel paths the duration of which are modeled by a six-sided die. The next activity after

216 these two paths merge in one path (gray nodes in Figure 1) will start only after the path with 217 the maximum duration is finished (the one with the maximum die cast value). If two dice are 218 cast, the maximum of both dice will not be 3.5 anymore, it will be higher. Analogously, if we 219 had more and more parallel paths (imagine 10 paths with one activity each, for instance), the 
220 probabilities of getting at least one six in one of the casts would be quite high. Therefore,

221 what is happening is that when several parallel paths converge, the mean project duration is

222 shifted to the right (the project ends later than expected) and the variance contracts.

223 Additionally, the skewness and kurtosis also momentarily deviate from the Normal

224 distribution (resembling more at that point an Extreme Value distribution). But unlike the

225 mean and the variance, they will dissipate again when more activities in the series are added

226 after the activities merger point or before the paths are separated.

227 This same effect can be visualized in the bottom half of Figure 1. The same two 228 distributions were chosen to illustrate how as more and more paths converge, the resulting 229 distribution (which actually is the highest order statistic of several distributions) shifts its 230 mean towards the right (it is increasingly higher than 5, which was the original mean duration value), whereas the dispersion (variance) also decreases (the distribution becomes more compressed). Overall, this phenomenon has been named merge bias or merge-event bias (Khamooshi and Cioffi 2013; Vanhoucke 2012), and it was the biggest problem that the original PERT had and the one that the scientific community has struggled most to solve. Indeed, this interesting phenomenon is hardly known by most professional construction schedulers nowadays.

The reason why this has been an enduring problem (indeed, no exact analytical solution has been found to date) is that despite apparently any distribution could be chosen to model the activity durations, that distribution would need to be sum-stable and max-stable. Sum-

240 stable means that the distribution of the convolution (sum) of several activity durations 241 (activities in series) should again belong to the same kind of distribution. An example is the 242 Normal distribution, in which, after being summed, the result is again Normal and with a mean and standard deviation as in equations 3 and 4. Max-stable means that after computing

244 the maximum from some distributions of the same type (activities in parallel), the result is 
again another distribution of the same type. The only two existing max-stable distributions are the Gumbel distribution and the Fréchet distribution, and neither one is sum-stable.

247 Hence, the ideal situation in which both operations can be performed with just one type of 248 distribution is not feasible.

To sum up, it is not possible to resort to an exact analytical approach for solving the PERT major shortcoming, and the only alternative is to work with mathematical approximations that keep the mean and variance relatively intact towards the end of the project. The logical alternative will be to resort to the Normal distribution, since at least it is sum-stable (which is the most frequent operation in any network due to the generally dominant number of activities in series), it is very well documented in the PERT-related literature, and it is still easy to handle.

Review of the most relevant PERT-related extensions

The study of project completion times when activities have uncertain durations mostly started attracting interest when PERT was published by Malcolm et al. (1959), but it has continued up to the present. Seminal works that followed PERT publication have been Anklesaria and Drezner's (1986) multivariate approach to estimating the project completion

262 time for stochastic networks, Elmaghraby's (1989) study, review and critique of the estimation of activity network parameters, Kamburowski's (1989) network analysis in

264 situations when probabilistic information is incomplete, and, more recently, the 265 computational studies by Ludwig et al. (2001) on bounding the makespan distribution of 266 stochastic project networks. These works are considered, without exception, classics in the 267 stochastic network analysis (SNA) field and have established the foundations of most of the later PERT-related research. 
However, computer-based (Monte Carlo) simulations have been to date probably the best alternative for obtaining a highly accurate representation of the actual project duration distribution no matter what the SNA topology and size are (e.g. (Douglas 1978; Hajdu 2013; Khamooshi and Cioffi 2013; Nelson et al. 2016)). Simulations will also be used here for comparing M-PERT outputs with the actual solution. But, obviously, the main reason why approximations like M-PERT are developed is because implementing computer simulations in schedule networks with special attributes (e.g., self-loops, alternative paths, correlated activities) is not usually straightforward for most construction students and practitioners (Ballesteros-Pérez et al. 2015). Also, manual procedures like M-PERT allow step-by-step incremental calculations, offering a more intuitive vision than simulation results.

Likewise, there have been other attempts to create pieces of software to develop PERTlike approaches for Stochastic Network Analysis (SNA) (e.g., Pontrandolfo (2000); Trietsch and Baker (2012)) that basically have the same aim as the Monte Carlo simulation, but allow a higher user interaction mostly oriented to enhancing the Decision Support process and progress monitoring, but with some accuracy loss.

In parallel, many recent algorithms (e.g., Mouhoub et al. (2011)) and other more advanced statistical techniques (Cho 2009; Węglarz et al. 2011), mostly involving Markov chains (e.g., Creemers et al. (2010); Magott and Skudlarski (1993); Xiangxing et al. (2010)), have also been developed for handling (optimizing) some project outcomes like activity timecost trade-offs (e.g., Azaron and Tavakkoli-Moghaddam (2007)), the total project duration (e.g., Baradaran et al. (2010)) or the project Net Present Value (NPV) (Creemers et al. 2010) while implementing PERT, but particularly in Resource-Constrained Project Schedules (e.g., Azaron et al. (2006); Baradaran et al. (2012); Yaghoubi et al. (2015)). These extensions, while worth noting, deal with computer implementations, unlike M-PERT, which adopts a manual approach. 
Furthermore, other techniques like fuzzy logic (e.g., Chen (2007)) and Artificial Neural

295 Network (ANN) analysis (e.g., Lu (2002)) have also been applied to improve activity

296 duration estimation and critical path(s) determination for later ranking purposes. We think

297 that these last two techniques and others in a similar fashion (e.g., Kuklan et al. (1993)),

298 despite having proved to be successful in other fields, do not offer a significant advantage

299 versus Monte Carlo simulations - commonly materialized in Schedule Risk Analysis (SRA)

300 (Vanhoucke 2012) - which are equally (or less) computer-intensive and give almost exact

301 results.

302 Also, concerning the monitoring and control dimension of PERT, some research has been

303 carried out to improve PERT for use as a project progress tool (e.g., Castro et al. (2007);

304 Wenying and Xiaojun (2011)), for example, by means of intersecting buffers after key 305 activities in networks considering both the time and cost dimensions (Khamooshi and Cioffi

306 2013), sometimes referred as Dynamic Planning and Scheduling (Azaron and Tavakkoli-

307 Moghaddam 2007; Yaghoubi et al. 2015). Also in this vein, some research has been

308 developed connected to crashing the PERT activities in order to fast-track project execution

309 (e.g., Abbasi and Mukattash (2001); Foldes and Soumis (1993)). The scope of these PERT

310 extensions, however, is related to control and schedule compression, which deviates from the

311 aim of the current paper (proposing a technique for estimating the total or remaining project

312 duration).

313 Interesting, but certainly minority research has also been devoted to studying the possible

314 effects of assuming independence versus the existence of (partial) correlation between

315 activities (e.g., Banerjee and Paul (2008); Cho (2009); Mehrotra et al. (1996); Sculli and

316 Shum (1991)). Some of their principles will be used here in M-PERT too, and two brief

317 examples are provided later in Figure 5. 
318 Concerning the application of PERT to situations where the activities are of a highly 319 repetitive nature, other PERT extensions have been published (e.g., RPERT (Aziz 2014)).

320 Repetition in activities will also be included among the M-PERT modelling capabilities later 321 by means of activities (or groups of activities) self-loops.

322 Concerning the number of point estimates to obtain the activity duration average and 323 standard deviation, as in expressions 1 and 2, extensive research has also been conducted. In 324 particular, the 'three-point estimates' from expressions 1 and 2, as this approach is commonly 325 known, make use of the minimum (optimistic), most likely (mode) and maximum 326 (pessimistic) durations that an activity duration following a Beta distribution can have. Other 327 attempts, which are actually as complementary to M-PERT as the original three-point 328 estimate proposal, have been proposed using two parameters (e.g., the mode with either the minimum or the maximum duration (Mohan et al. 2006)), three parameters but with some degree of reparametrization (e.g., Herrerías-Velasco et al. 2011), or even up to four parameters (e.g., (Hahn 2008)). However, normally, when the parameters have been changed, the distribution type modelling the activity duration changes too.

Another minor but relevant subfield of research to this study is the work that deals with the PERT network reducibility problem. Essentially, M-PERT is a technique that recursively downsizes the schedule network by merging activities in series and in parallel. With a similar aim, but with a strong emphasis on the computational point of view, the original works by Ringer (1969), Elmaghraby (1989) and Bein et al. (1992) studied this challenging problem in extensive detail. As M-PERT lends itself to a manual reduction approach, the reader interested in computer implementations is referred to these remarkable works.

340 Finally, as was anticipated earlier, an overwhelming amount of research has been devoted 341 to trying to improve the original Beta distribution fitness (Hahn 2008; Premachandra 2001), 342 or just try to find another probability distribution that better fits the activity durations (e.g., 
343 Lau and Somarajan (1995); López Martín et al. (2012)). The variety of distributions that can

344 be found as an alternative to the Beta distribution is overwhelming. They include the doubly

345 truncated normal distribution (Kotiah and Wallace 1973), the triangular distribution (Johnson 346 1997), the log-normal distribution (Mohan et al. 2006), the mixed beta and uniform 347 distribution (Hahn 2008), and the Parkinson distribution (Trietsch et al. 2012), to cite a few.

348 As expected, however, this line of research has caused quite a lot of controversy, with 349 some researchers in favor of pursuing the fit-for-all distribution (e.g., Clark (1962); Golenko350 Ginzburg (1989); Grubbs (1962); Healy (1961); Sasieni (1986)) and others against (e.g., 351 Herrerías-Velasco et al. (2011); Kamburowski (1997); Pleguezuelo et al. (2003)). As 352 MacCrimmon and Ryavec (1964) anticipated and we again proved in Figure 1, the particular 353 distribution is hardly relevant, so no more references will be made to this discussion.

354 With the most relevant PERT-related lines of research identified, it is time to identify the subset of works upon which M-PERT has been built, which are those that deal with the merge event bias in some way or another. These works are summarized in Table 1 by columns, and all of them are approximate techniques for solving some flaws that the original PERT technique had. Unfortunately, these works are not widely known by the research community. Even more surprisingly, most of them were unaware of the others, even though all but the first one were contemporaneous. For the sake of brevity, some details have been given by the rows in Table 1 and some more will be specified later in the M-PERT outline section.

Of particular interest perhaps, is the work by Pritsker (1966), who developed the

365 Graphical Evaluation and Review Technique (GERT), which would have fulfilled the 366 promise of devising a new PERT without most of its problems if it had not been for the 
mathematical complexity that did not allow the authors to finally implement it without resorting to Monte Carlo simulations. However, this technique introduced very interesting features, some of which M-PERT has inherited, like self-loops and probabilistic (alternative) paths.

Last of all, another small subset of approximate PERT variants sought to tackle the merge bias problem by obtaining the upper and/or lower bounds of the project total duration. Without seeking to be exhaustive, maybe some of the most relevant findings were the Clark's (one of the PERT creators) bias correction procedure (Clark 1961, 1962), the ' $f$ ' estimate (Fulkerson 1962) and the Modified Network Evaluation Technique (PNET) (Ang et al. 1975). A recent exhaustive study by González et al. (2014) comparing the accuracy achieved by these methods when approximating the Project duration mean and variance in networks with a varied array of topological indicators showed that the best methods were the ' $\mathrm{f}$ ' estimate for the mean (with an average error of 2.6\%) and the PNET for the variance (with an average error of $29.8 \%$ ). Anticipating some results presented later, the methods summarized in Table 1, on which M-PERT was built, exhibited smaller errors in the benchmark networks tested, which has allowed M-PERT to currently have average errors below $2 \%$ for the mean and below $10 \%$ for the variance. Therefore, these methods, despite deserving acknowledgment, will not be referred to any more.

\section{M-PERT outline}

M-PERT has been built upon the five models presented in Table 1, most of which shared some common traits (evidenced in lines with several ' $\checkmark$ ' marks). All five methods made use of Activity-on-Arc (AoA) networks, whereas M-PERT resorts to Activity-on-Node (AoN) representation since nowadays it is more user-friendly for practitioners and more commonly

391 found in software (e.g., Microsoft Project, Oracle Primavera). 
Consequently, the precedence relationships are necessarily represented by means of the arrows connecting the nodes. Initially, it is well known that, given two activities $i$ and $j$, there can be four precedence relationships: Finishes $i-$ Starts $j$ (the most common by far), Starts $i-$ Starts $j$, Finishes $i$-Finishes $j$, and Starts $i$-Finishes $j$, all of them with or without time lags between them.

Representation of the last three, despite being possible, significantly complicates the network visualization and the critical path analysis when the number of activities is high since some arrows depart the nodes from their back and/or reach the nodes at their front. Fortunately, as recently found by Lu and Lam (2009), these three precedence types can be reformulated as a Finish-Start relationship, as Figure 2 illustrates, and with which scheduling students can be trained. Hence, from now on, M-PERT will make exclusive use of AoN networks with Finish-Start (FS) precedence relationships, and should the user wish to establish different types of precedence, he/she should refer to Figure 2, or, alternatively, to Lu and Lam's (2009) comprehensive treatment of non-finish-to-start relationships in project networks, and transform them to FS relationships.

\section{$<$ Figure 2>}

Essentially, M-PERT is a reduction technique in which project activities are merged by groups of two or more, resulting in a new single merged activity, and this process is repeated until there is just one activity left, which represents the total project duration. An idea of this sequential merging procedure can be observed later in Figure 4 and the supplemental data. The merging procedure was proposed by Cox (1995), and it is an intuitive and relatively quick approach for reducing any (complex) network into a simple one. Of course, the challenge is how to exactly merge different activities (which in the end correspond to

415 duration distributions). For that purpose and as justified earlier, it is assumed that the activity 416 durations follow a Normal distribution. Being aware that the specific probability distribution 
417 was hardly important, the Normal assumption was also made by three out of the five methods

418 stated in Table 1, and this obeys the need of simplifying as much as possible the merging

419 operation of activities in series, which is the most frequent in any network. The merger

420 operations in the serial activities are represented in the first row of Figure 3.

$<$ Figure 3 $>$

422

Figure 3 is, in general, quite self-explanatory and basically represents the most common operations (mergers) that can be found in any network - mostly serial activities (first row) and maxima of multiple paths (bottom row) - along with other interesting operations that GERT (Pritsker 1966) proposed (probabilistic paths, self-loops and activity minima) but was not able to implement without ad hoc computational programmes. For illustrative purposes, the case study shown later in Figure 4 will provide examples of these logic operations.

Particularly in construction projects, operations like probabilistic (alternative) paths might seem quite straightforward for modelling uncertain courses of action that will mostly depend on information that is still unknown, or at least inaccurate, at the conceptual stage. Examples are the choice between different types of foundations depending on the ground conditions or the soil-bearing capacity, or just the need to take special measures if the project uncovers archeological remains.

434 Self-loops, on the other hand, are helpful for representing activities being repeated after an unsuccessful outcome. These activities can correspond to isolated activities (e.g., a load test, a calibration) or even groups of activities (e.g., a whole project drawing up process, a 437 series of defective structural elements, a non-compliant subnetwork within a water supply system). It is up to the scheduler, however, to decide how many loops, or even how many multiple nested loops, could be feasibly implemented before the construction manager or the 440 client stops the iterations. 
Similarly, activity minima, despite certainly being much less frequent than activity

maxima, can also be useful at times to allow the project continuation after one (or some) of

443 the predecessors are finished. A distinctive quality of activity minima versus probabilistic

444 paths is that all the activities involved in the minima merger must be executed at some point

445 before the project finishes, whereas in probabilistic paths only one (or a subset of) path(s)

446 will be finally carried out. Examples of activity minima are the supply of any

447 electromechanical equipment among a series of any other equipment that needs to be

448 received in a worksite before the mechanical engineers can start working, or the clearance of

449 any stocking areas of a linear worksite before a new pipeline section can be supplied.

450 Overall, for every operation by rows in Figure 3, their representation (second column) is

451 shown; the input parameters (third column) necessary for defining the activities and

452 performing the merger into a single activity (which is generically named $k$ ) are provided; the

453 output parameters (fourth column) that define the new duration mean and variance of the

454 resulting merged activity $k$ are provided; a representation of the result (fifth column) is

455 shown; and, finally, some observations (last column on the right) that clarify how to apply the

456 merger operations in particular (more complex) contexts.

457 It is worth highlighting that M-PERT requires that the activity duration means $\left(\mu_{i}\right)$ and

458 variances $\left(\sigma_{i}\right)$ have been specified for each activity beforehand, a requirement that forces the

459 student or scheduler to elicit them or, if he/she wanted to start from the three-point estimates

$460(O, L$ and $P)$, it would require a first stage of application of equations 1 and 2 (with the

461 correction proposed in equation 6) for each activity, like the original PERT.

462 Concerning the origin of the mathematical expressions stated for all the mergers (stated in

463 the Output parameters column), the serial activities merger just corresponds to the sum of

464 means and variances of several Normal distributions (as in equations 3 and 4), whereas the 
the mathematical expressions of the mixture (Union) of $n$ non-overlapping (Normal) population samples $\left(X_{i}=X_{1} \ldots X_{n}\right)$, which are:

$$
{ }_{k}=\frac{{ }_{i} W_{X_{i}} X_{i}}{{ }_{i} W_{X_{i}}}
$$

$$
{ }_{k}^{2}=\frac{{ }_{i} W_{X_{i}}{ }_{X_{i}}^{2}}{{ }_{i} W_{X_{i}}}+\frac{{ }_{i<j} W_{X_{i}} W_{X_{j}}\left({ }_{X_{i}}{ }_{X_{j}}\right)^{2}}{\left({ }_{i} W_{X_{i}}\right)^{2}}
$$

$$
\text { Where the } W_{X i} \text { are the weights of each Normal population merged, that is, the proportion }
$$

by which that Normal distribution (representing a set of previously merged activities'

duration) will be present in the resulting distribution after the next merger. For example, for the probabilistic paths, the weights are the probabilities of each path occurring, that is, $W_{X I}=p$ and $W_{X 2}=1-p$ for two paths; or $W_{X i}=p_{x}$ for $i=x=1,2, \ldots n$ paths, with ${ }_{x=1}^{n} W_{x}=1$ always. For the self-loops, an activity $i$ is merged with itself - activity $i$ 's mean $\mu_{X_{l}}=\mu_{i}$ and standard deviation $\sigma_{X_{1}}=\sigma_{i}$ when the activity happens just once with probability $W_{X I}=1-p_{i}$, and $\mu_{X_{2}}=2 \mu_{i}$ and $\sigma_{X_{2}}=\sqrt{2}$ $\sigma_{i}$ when it is repeated with probability $W_{X 2}=p_{i}$.

Finally, the case of the maximum from two Normal distributions (the durations of two activities) was coincidentally proposed by Clark (1961) but at that time it required intensive use of tables and did not allow for correlation $(\rho)$ between both activities. In particular, the expression for the maximum was taken from Sculli and Shum (1991) and was also used by Cox (1995), whereas the expression for the minimum was taken from Nadarajah and Kotz (2008). Both expressions, despite looking long, are very easy to calculate.

$$
\text { The obvious problem with the maxima and minima from several activities is that the }
$$
formulae only allow for the merger of two of them at the same time. Then these expressions can be applied recursively until there is just one activity left, a moment in which the activity will be in series again and can keep being merged with its neighbor activities in series. This recursive merging procedure is not new either, as it was successfully applied by Cox (1995), 
489 Gong and Hugsted (1993) and Sculli and Shum (1991). However, it is also true that when

490 more than two activities are merged, there is a small error in the final mean and variance

491 calculation, which is also dependent (but to a minimum extent) on the exact merger order too.

492 Simulations performed that try to estimate the maximum error magnitude with M-PERT

493 identified how this error is maximized when the different paths' duration mean is the same,

494 and variances are also identical between the parallel paths. However, even in that case, the

495 error obtained when merging "eight" activities under the same node (which is a higher

496 number than most of the real projects have under one converging node) causes an error of

$4976.7 \%$ in the mean and $7.3 \%$ in the standard deviation, which are considered, in general, good

498 approximations.

499 Therefore, M-PERT consists of merging activities until there is one activity left that

500 models the total project duration by means of $\mu_{p}$ and $\sigma_{p}$, which correspond to the project

501 duration mean and standard deviation, respectively. The order in which merger operations are

502 performed is relevant too. For example, if a self-loop comprised several activities, those inner

503 activities should first be merged, and then the self-loop resolved; or, if there are several

504 activities in series within a parallel path, those activities need to be merged before the path

505 can be merged with other paths. Overall, there must be an overriding use of common sense

506 when performing the mergers, and unless there is correlation among activities (a situation that

507 will be treated separately later), the merging procedure is quite straightforward.

508 There is just one last step left in the application of M-PERT. Obviously, the resulting

509 distribution modelling the total project duration is forced to be Normal in M-PERT, but, in

510 reality, this distribution might be more similar to an Extreme Value distribution when there is

511 a "high number of parallel paths converging all of them to the same node (activity)". This is

512 not a secret, but it was not properly incorporated in a PERT model until Dodin and Sirvanci 
513 (1990). He proposed using the Gumbel distribution (despite it being generically named the

514 Extreme Value distribution) for modelling the total project duration.

515 In M-PERT, however, we think the Normal distribution constitutes a reasonable

516 approximation. This is because most construction projects, despite usually including multiple

517 paths, they do not "all converge in the same single activity", rather they have multiple paths

518 which converge at different activities. In other words, there is not a dominance of maxima

519 computation, but some maxima calculations mixed with a high number of activities in series

520 or activity maxima in series, both of which quickly degenerate (as shown in Figure 1) in

521 Normal distributions. Furthermore, students who are exposed to learning M-PERT are much

522 more familiar with the Normal distribution than with Extreme Value distributions.

523

524 Case study

$525 \quad$ A fictitious and simplified bridge project

526 M-PERT can be equally applied to simple and complex networks, the only difference

527 being the number of mergers that are required to be performed before the network is reduced

528 to a single activity representing the project duration. In this regard, Figure 4 presents the

529 application in a simple but representative bridge project construction with three piles (three

530 parallel paths), in which, purposefully, all possible kinds of merging operations have been

531 included. The activities 'Start' and 'End' are just dummy activities (null duration).

On the lower part of Figure 4, the sequential merging procedure has been exemplified.

534 Note, however, that the activities represent the standard deviation, not the variance, and this

535 is to be considered when applying all the expressions stated in Figure 3. Furthermore, the

536 type of merger operation has been stated in the five-step (solution) reduction procedure,

537 replacing the original activity descriptions and allowing the reader to understand where each 
newly merged activity came from. All these indications however, are not necessary when we try to expedite the manual calculation process.

Few explanations are needed at this stage, since the merger procedure just requires the application of simple mathematical expressions already introduced mostly in Figure 3. It is worth highlighting that, unlike the original PERT with fixed paths (in which what was described in the network had to be necessarily carried out and excluded the possibility of going backwards), the introduction of probabilistic paths, minima and self-loops allows for a closer representation of construction projects. In this sense, M-PERT allows for some paths happening while others do not, and that a group (or even the whole project if need be) is repeated thanks to the inclusion of a self-loop. Overall, the modelling possibilities of MPERT are significantly more powerful than the original PERT.

\section{$\underline{\text { Networks with correlation between activities }}$}

Sometimes there are networks (project schedules) in which the merger procedure ends in a knot that cannot be untangled. This is quite usual indeed when there are several parallel paths that do not depart from the same node and/or do not converge in the same node (Mehrotra et al. 1996). In those situations, the partially reduced network can end up in (sub)networks similar to the ones shown in Figure 5, although maybe with more nodes.

\section{$<$ Figure 5 $>$}

For solving these subnetworks that usually represent a small portion of the whole network, authors like Mehrotra et al. (1996) and Sculli and Shum (1991) proposed a sequential recursive procedure that involves calculating all the variance and covariance values between the 'untangled' paths. Implementing this procedure basically requires identifying all the possible paths and then merging them, first, two of them, and then one by 
562 one with the already merged group. However, the user needs to be aware that there is a correlation $(\rho \neq 0)$ between activities when applying the maxima (or minima) expressions from

564 Figure 3, that is, between paths that have some activities in common (as the covariance 565 between those two paths will not generally be zero).

As can be seen in Figure 5, identifying all the possible paths is not difficult unless the network knot size involves a lot of activities and is very dense in precedence relationships (number of arrows). The only tricky part is to calculate the correct value of $\rho$ between the paths. However, Sculli and Shum (1991) derived the generic expression for obtaining it, but since that expression is somehow difficult to understand for the beginner, the $\rho$ expressions for up to four parallel paths (normally ordered from higher to lower mean duration $\mu_{k}$ and sequentially named $A, B, C$ and $D$ ) are given here:

$$
\begin{aligned}
& { }_{A B}=\frac{C O V_{A B}}{A \quad B} \\
& { }_{A B C}=\frac{C O V_{A C} \Phi\left({ }_{A B}\right)+C O V_{B C}\left(1 \quad \Phi\left({ }_{A B}\right)\right)}{{ }_{A B} \quad C}
\end{aligned}
$$

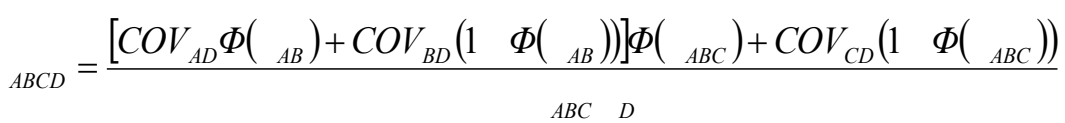

Where $\rho_{A B}, \rho_{A B C}, \rho_{A B C D}$ representing, respectively, the correlation coefficients as paths A with $\mathrm{B}, \mathrm{AB}$ with $\mathrm{C}$, and $\mathrm{ABC}$ with $\mathrm{D}$ are merged one by one with the previously merged paths. $C O V_{X Y}$ simply corresponds to the sum of variances of the activities that paths $X$ and $Y$ have in common, and $\sigma_{X}$ and $\sigma_{Y}$, the standard deviations of the paths $X$ and $Y$ respectively, with $X, Y=A, B, C, D$. Examples of calculation of the variance and covariance values have been provided in Figure 5 in the covariance matrices.

Finally, for the sake of clarity, the complete step-by-step procedure for implementing expressions 9 to 11 in a project network with four paths with correlation has been described 
in the supplemental data under Alternative 2 in the example project 0 , along with other multiple examples of M-PERT exercises.

586

\section{Results}

The five methods from which M-PERT has been built were tested in several benchmark networks. There is no reason to believe that M-PERT, which makes use of their most meritorious contributions, even including new features for better modelling real-life projects, will have a lower accuracy. Anyhow, M-PERT has been applied to one network in this paper and its accuracy comparison is presented in Figure 6.

It must be borne in mind, though, that Figure 6 shows a comparison of four statistical distributions of which the distribution parameters are perfectly known, not a comparison of datasets against another dataset or a known distribution, as is more usual in research analyses. Hence, we will not be testing how 'significant' the difference between any two distributions is, as it is already known that they are different distributions. Instead, a graphical approach is adopted here for illustrating the location and dispersion deviations between the distributions. exact solution of the project duration distribution (obtained through computer simulations) by modelling the activity durations with Normal distributions and Uniform distributions respectively. As stated before, the choice of one or other distribution hardly has any

604 relevance, an outcome proven again by observing how close both gray curves are. The average (project duration) of these two simulated distributions is close to 147.5 days despite they being lightly positively skewed and leptokurtic.

607 The dashed curve, on the other hand, represents the approximation offered by M-PERT by using the Normal distribution modelling the project duration distribution, whose 
609 parameters were obtained at the bottom of Figure 4. The bridge project, with three dominant

610 parallel paths, does not deviate much from the Normal approximation. It is also easy to

611 appreciate how the M-PERT project duration mean is close to 147 days (estimation error

612 below 1\% when compared to the simulated curves, whereas the variance or dispersion error is

613 also below 10\%).

614 Last of all, the continuous black curve representing the classical PERT approximation

615 significantly falls behind, with an average project duration of 133 days (error above 10\%).

616 Again, this evidences how inaccurate the original PERT is when there are parallel paths and

617 the merge event bias is neglected.

618 Finally, for reassurance, results in other networks exemplifying step-by-step calculations,

619 along with the (simulated) actual project duration distribution, can be found as supplemental 620 data.

\section{Discussion and conclusions}

623 A new scheduling technique named M-PERT has been proposed. This technique takes

624 advantage of several methods that were proposed since PERT was devised for correcting several shortcomings that the original technique had. In particular, in situations with multiple parallel paths (the norm in construction projects), the original PERT resulted in a project duration underestimation and a variance overestimation.

A systematic literature review has been developed in order to justify why M-PERT concentrates on the really significant weaknesses of PERT and why, by neglecting other accessorial aspects, the resulting tool is still accurate, but not complex. Indeed, the method proposed is easy to implement and easy to learn due to its intuitive nature and simplifying assumptions. This makes M-PERT an attractive tool for teaching scheduling basics to construction and project management students, especially since its calculations can be 
developed manually or by means of very simple spreadsheets. Finally, an application has been included in which the new features offered by M-PERT, such as the minima of activities, probabilistic (alternative) paths or activity or groups of activities self-loops evidence a higher resemblance between scheduled networks and real construction projects.

However, there is still a long way to go. Indeed, despite this tool really departing from the original PERT conception, it is still open to improvement. The cost dimension has not been included in M-PERT, but it should follow a very similar approach. However, the inclusion of resources in the analysis really represents a challenging route that should probably be incorporated in the next versions of this scheduling technique.

\section{Data availability}

All data generated or analyzed during the study are included in the submitted article or supplemental materials files.

\section{References}

649

650

651

652

653

654

655

656

657

658

659

660

661

662

663

664

665

Abbasi, G. Y., and Mukattash, A. M. (2001). "Crashing PERT networks using mathematical programming." International Journal of Project Management, 19(3), 181-188.

Ang, A. H.-S., Chaker, A. A., and Abdelnour, J. (1975). "Analysis of Activity Networks under Uncertainty." Journal of the Engineering Mechanics Division, 101(4), 373-387.

Anklesaria, K. P., and Drezner, Z. (1986). "A Multivariate Approach to Estimating the Completion Time for PERT Networks." Journal of the Operational Research Society, 37(8), 811-815.

Asmar, M. El, Hanna, A. S., and Whited, G. C. (2011). "New Approach to Developing Conceptual Cost Estimates for Highway Projects." Journal of Construction Engineering and Management, American Society of Civil Engineers, 137(11), 942-949.

Azaron, A., Katagiri, H., Sakawa, M., Kato, K., and Memariani, A. (2006). "A multiobjective resource allocation problem in PERT networks." European Journal of Operational Research, 172(3), 838-854.

Azaron, A., and Tavakkoli-Moghaddam, R. (2007). "Multi-objective time-cost trade-off in dynamic PERT networks using an interactive approach." European Journal of Operational Research, 180(3), 1186-1200.

Aziz, R. F. (2014). “RPERT: Repetitive-Projects Evaluation and Review Technique.” 

Alexandria Engineering Journal, 53(1), 81-93.

Ballesteros-Pérez, P., Skitmore, M., Das, R., and del Campo-Hitschfeld, M. L. (2015). "Quick Abnormal-Bid-Detection Method for Construction Contract Auctions." Journal of Construction Engineering and Management, 141(7), 4015010.

Banerjee, A., and Paul, A. (2008). "On path correlation and PERT bias." European Journal of Operational Research, 189(3), 1208-1216.

Baradaran, S., Fatemi Ghomi, S. M. T., Mobini, M., and Hashemin, S. S. (2010). "A hybrid scatter search approach for resource-constrained project scheduling problem in PERTtype networks." Advances in Engineering Software, 41(7-8), 966-975.

Baradaran, S., Fatemi Ghomi, S. M. T., Ranjbar, M., and Hashemin, S. S. (2012). "Multimode renewable resource-constrained allocation in PERT networks." Applied Soft Computing, 12(1), 82-90.

Bein, W. W., Kamburowski, J., and Stallmann, M. F. M. (1992). "Optimal Reduction of Two-Terminal Directed Acyclic Graphs.” SIAM Journal on Computing, 21(6), 11121129.

Castro, J., Gómez, D., and Tejada, J. (2007). “A project game for PERT networks.” Operations Research Letters, 35(6), 791-798.

Chen, S.-P. (2007). "Analysis of critical paths in a project network with fuzzy activity times." European Journal of Operational Research, 183(1), 442-459.

Cho, S. (2009). "A linear Bayesian stochastic approximation to update project duration estimates.” European Journal of Operational Research, 196(2), 585-593.

Clark, C. E. (1961). "The Greatest of a Finite Set of Random Variables." Operations Research, INFORMS, 9(2), 145-162.

Clark, C. E. (1962). "Letter to the Editor-The PERT Model for the Distribution of an Activity Time." Operations Research, INFORMS, 10(3), 405-406.

Cox, M. (1995). "Simple normal approximation to the completion time distribution for a PERT network.” International Journal of Project Management, 13(4), 265-270.

Creemers, S., Leus, R., and Lambrecht, M. (2010). "Scheduling Markovian PERT networks to maximize the net present value." Operations Research Letters, 38(1), 51-56.

Dodin, B., and Sirvanci, M. (1990). "Stochastic networks and the extreme value distribution." Computers \& Operations Research, 17(4), 397-409.

Douglas, D. E. (1978). "PERT and simulation." WSC '78 Proceedings of the 10th conference on Winter simulation, IEEE, 89-98.

Elmaghraby, S. E. (1989). "The estimation of some network parameters in the PERT model of activity networks: review and critique." Advances in Project Scheduling (eds. Slowinski \& Weglarz), Elsevier, 371-432.

Foldes, S., and Soumis, F. (1993). "PERT and crashing revisited: Mathematical generalizations.” European Journal of Operational Research, 64(2), 286-294.

Fulkerson, D. R. (1962). "Expected Critical Path Lengths in PERT Networks.” Operations Research, 10(6), 808-817.

Golenko-Ginzburg, D. (1989). "PERT assumptions revisited.” Omega, 17(4), 393-396. 
Gong, D., and Hugsted, R. (1993). "Time-uncertainty analysis in project networks with a new merge-event time-estimation technique." International Journal of Project Management, 11(3), 165-173.

González, G. E. G., Hernández, G. O., and Roldán, F. (2014). “Effect of Network’s Morphology and Merge Bias Correction Procedures on Project Duration Mean and Variance." Procedia - Social and Behavioral Sciences, 119, 2-11.

Grubbs, F. E. (1962). “Attempts to Validate Certain PERT Statistics or 'Picking on PERT.”' Operations Research, 10(6), 912-915.

Hahn, E. D. (2008). "Mixture densities for project management activity times: A robust approach to PERT.” European Journal of Operational Research, 188(2), 450-459.

Hajdu, M. (2013). "Effects of the application of activity calendars on the distribution of project duration in PERT networks." Automation in Construction, Elsevier B.V., 35, 397-404.

Hajdu, M., and Bokor, O. (2014). "The Effects of Different Activity Distributions on Project Duration in PERT Networks.” Procedia - Social and Behavioral Sciences, 119, 766775.

Healy, T. L. (1961). “Activity Subdivision and PERT Probability Statements.” Operations Research, INFORMS, 9(3), 341-348.

Herrerías-Velasco, J. M., Herrerías-Pleguezuelo, R., and Van Dorp, J. R. (2011). "Revisiting the PERT mean and variance." European Journal of Operational Research, 210(2), $448-451$.

Johnson, D. (1997). "The Triangular Distribution as a Proxy for the Beta Distribution in Risk Analysis." Journal of the Royal Statistical Society: Series D (The Statistician), 46(3), 387-398.

Kamburowski, J. (1989). "PERT networks under incomplete probabilistic information." Advances in Project Scheduling (eds. Slowinski \& Weglarz), Elsevier, 433-466.

Kamburowski, J. (1997). “New validations of PERT Times.” Omega, 25(3), 323-328.

Khamooshi, H., and Cioffi, D. F. (2013). "Uncertainty in Task Duration and Cost Estimates: Fusion of Probabilistic Forecasts and Deterministic Scheduling." Journal of Construction Engineering and Management, American Society of Civil Engineers, 139(5), 488-497.

Kotiah, T. C. T., and Wallace, N. D. (1973). "Another Look at the PERT Assumptions." Management Science, INFORMS, 20(1), 44-49.

Kuklan, H., Erdem, E., Nasri, F., and Paknejad, M. J. (1993). "Project planning and control: an enhanced PERT network." International Journal of Project Management, 11(2), 8792.

Lau, H.-S., and Somarajan, C. (1995). “A proposal on improved procedures for estimating task-time distributions in PERT." European Journal of Operational Research, 85(1), 39-52.

López Martín, M. M., García García, C. B., García Pérez, J., and Sánchez Granero, M. A. (2012). "An alternative for robust estimation in Project Management." European Journal of Operational Research, 220(2), 443-451. 
Lu, M. (2002). "Enhancing Project Evaluation and Review Technique Simulation through Artificial Neural Network-based Input Modeling." Journal of Construction Engineering and Management, American Society of Civil Engineers, 128(5), 438-445.

Lu, M., and Lam, H.-C. (2009). "Transform Schemes Applied on Non-Finish-to-Start Logical Relationships in Project Network Diagrams." Journal of Construction Engineering and Management, 135(9), 863-873.

Ludwig, A., Möhring, R. H., and Stork, F. (2001). "A Computational Study on Bounding the Makespan.” Annals of Operations Research, 102, 49-64.

MacCrimmon, K. R., and Ryavec, C. A. (1964). "An Analytical Study of the PERT Assumptions." Operations Research, 12(1), 16-37.

Magott, J., and Skudlarski, K. (1993). "Estimating the mean completion time of PERT networks with exponentially distributed durations of activities." European Journal of Operational Research, 71(1), 70-79.

Malcolm, D. G., Roseboom, J. H., Clark, C. E., and Fazar, W. (1959). “Application of a Technique for Research and Development Program Evaluation." Operations Research, 7(5), 646-669.

Mehrotra, K., Chai, J., and Pillutla, S. (1996). "A study of approximating the moments of the job completion time in PERT networks." Journal of Operations Management, 14(3), $277-289$.

Mohan, S., Gopalakrishnan, M., Balasubramanian, H., and Chandrashekar, A. (2006). “A lognormal approximation of activity duration in PERT using two time estimates." Journal of the Operational Research Society, 58(6), 827-831.

Mouhoub, N. E., Benhocine, A., and Belouadah, H. (2011). "A new method for constructing a minimal PERT network.” Applied Mathematical Modelling, Elsevier Inc., 35(9), 4575-4588.

Mummolo, G. (1997). "Measuring uncertainty and criticality in network planning by PERTpath technique." International Journal of Project Management, 15(6), 377-387.

Nadarajah, S., and Kotz, S. (2008). "Exact distribution of the max/min of two Gaussian random variables." IEEE Transactions on Very Large Scale Integration (VLSI) Systems, $16(2), 210-212$.

Nelson, R. G., Azaron, A., and Aref, S. (2016). "The use of a GERT based method to model concurrent product development processes." European Journal of Operational Research, 250(2), 566-578.

Pleguezuelo, R. H., Pérez, J. G., and Rambaud, S. C. (2003). "A note on the reasonableness of PERT hypotheses." Operations Research Letters, 31(1), 60-62.

PMI. (1996). A Guide to the Project Management Body of Knowledge. 1st edition. Project Management Institute. Newton square, USA.

PMI. (2008). A Guide to the Project Management Body of Knowledge. 5th edition. (P. M. I. N. square. USA, ed.), Project Management Institute. Newton square, USA.

Pontrandolfo, P. (2000). "Project duration in stochastic networks by the PERT-path technique." International Journal of Project Management, 18, 215-222.

Premachandra, I. M. (2001). "An approximate of the activity duration distribution in PERT." 
792

793

794

795

796

797

798

799

800

801

802

803

804

805

806

807

808

809

810

811

812

813

814

815

816

817

818

819

820

821

822

823

824

825

826

827

Pritsker, A. A. B. (1966). GERT: Graphical Evaluation and Review Technique. RM-4973NASA. National Aeronautics and Space Administration under Contract No. NASr-21. Retrieved 2006-12-05.

Ringer, L. J. (1969). "Numerical Operators for Statistical Pert Critical Path Analysis." Management Science, 16(2), 136-143.

Sasieni, M. W. (1986). "Note-A Note on Pert Times.” Management Science, INFORMS, 32(12), 1652-1653.

Sculli, D., and Shum, Y. W. (1991). "An approximate solution to the pert problem." Computers \& Mathematics with Applications, 21(8), 1-7.

Stauber, B. R., Douty, H. M., Fazar, W., Jordan, R. H., Weinfeld, W., and Manvel, A. D. (1959). "Federal Statistical Activities." The American Statistician, 13(2), 9-12.

Tatterson, J. (1974). "PERT, CPM and the export process.” Omega, 2(3), 421-426.

Tavares, L. V. (2002). “A review of the contribution of Operational Research to Project Management.” European Journal of Operational Research, 136(1), 1-18.

Trietsch, D., and Baker, K. R. (2012). "PERT 21: Fitting PERT/CPM for use in the 21st century." International Journal of Project Management, APM and IPMA and Elsevier Ltd, 30(4), 490-502.

Trietsch, D., Mazmanyan, L., Gevorgyan, L., and Baker, K. R. (2012). "Modeling activity times by the Parkinson distribution with a lognormal core: Theory and validation." European Journal of Operational Research, Elsevier B.V., 216(2), 386-396.

Vanhoucke, M. (2012). Project Management with Dynamic Scheduling. Springer Berlin Heidelberg, Berlin, Heidelberg.

Węglarz, J., Józefowska, J., Mika, M., and Waligóra, G. (2011). "Project scheduling with finite or infinite number of activity processing modes - A survey." European Journal of Operational Research, 208(3), 177-205.

Wenying, L., and Xiaojun, L. (2011). "Progress Risk Assessment for Spliced Network of Engineering Project Based on Improved PERT.” Systems Engineering Procedia, 1, 271278.

Woolf, C. ., Cass, W., and McElroy, J. (1968). "The use of 'Program Evaluation and Review Technique' (PERT) in the design and control of a medical research project." Computers and Biomedical Research, 2(2), 176-186.

Xiangxing, K., Xuan, Z., and Zhenting, H. (2010). "Markov skeleton process in PERT networks." Acta Mathematica Scientia, 30(5), 1440-1448.

Yaghoubi, S., Noori, S., Azaron, A., and Fynes, B. (2015). "Resource allocation in multiclass dynamic PERT networks with finite capacity." European Journal of Operational Research, Elsevier B.V., 247(3), 879-894. 


\begin{tabular}{|c|c|c|c|c|c|}
\hline$\nabla$ Highlights & $\begin{array}{c}\text { Pritsker } \\
\text { (1966) }\end{array}$ & $\begin{array}{c}\text { Sculli and } \\
\text { Shum } \\
(1991)\end{array}$ & $\begin{array}{l}\text { Gong \& } \\
\text { Hugsted } \\
(1993)\end{array}$ & $\begin{array}{c}\text { Cox } \\
(1995)\end{array}$ & $\begin{array}{l}\text { Mehrotra } \\
\text { et al. } \\
(1996)\end{array}$ \\
\hline $\begin{array}{l}\text { Avoids having to calculate all the } \\
\text { possible paths (critical and non- } \\
\text { critical) (allows big networks) }\end{array}$ & $\checkmark$ & & $\checkmark$ & $\checkmark$ & \\
\hline $\begin{array}{l}\text { Allows for recursive application of } \\
\text { activity maxima and/or minima (time- } \\
\text { saving simplification) }\end{array}$ & & $\checkmark$ & $\checkmark$ & $\checkmark$ & \\
\hline $\begin{array}{c}\text { Normal distributions for modelling the } \\
\text { activity durations } \\
\text { (only mean and variance matter) }\end{array}$ & & $\checkmark$ & $\checkmark$ & & $\checkmark$ \\
\hline $\begin{array}{c}\text { Allows for correlation between } \\
\text { different activities } \\
\text { (allows complex projects) }\end{array}$ & $\checkmark$ & $\checkmark$ & $\checkmark$ & & $\checkmark$ \\
\hline $\begin{array}{c}\text { Use of simple expressions for solving } \\
\text { the activity mergers } \\
\text { (calculation simplicity) }\end{array}$ & & & & $\checkmark$ & $\checkmark$ \\
\hline $\begin{array}{l}\text { Allows for extra features (activity } \\
\text { minima, self-loops or probabilistic } \\
\text { paths, etc) (resemblance to reality) }\end{array}$ & $\checkmark$ & & & & \\
\hline $\begin{array}{c}\text { Number of benchmark networks in } \\
\text { which the model was tested (Number } \\
\text { of validation tests) }\end{array}$ & 4 & 2 & 3 & 2 & 10 \\
\hline
\end{tabular}

Table 1. Key works on which PERT 2.0 has been based 


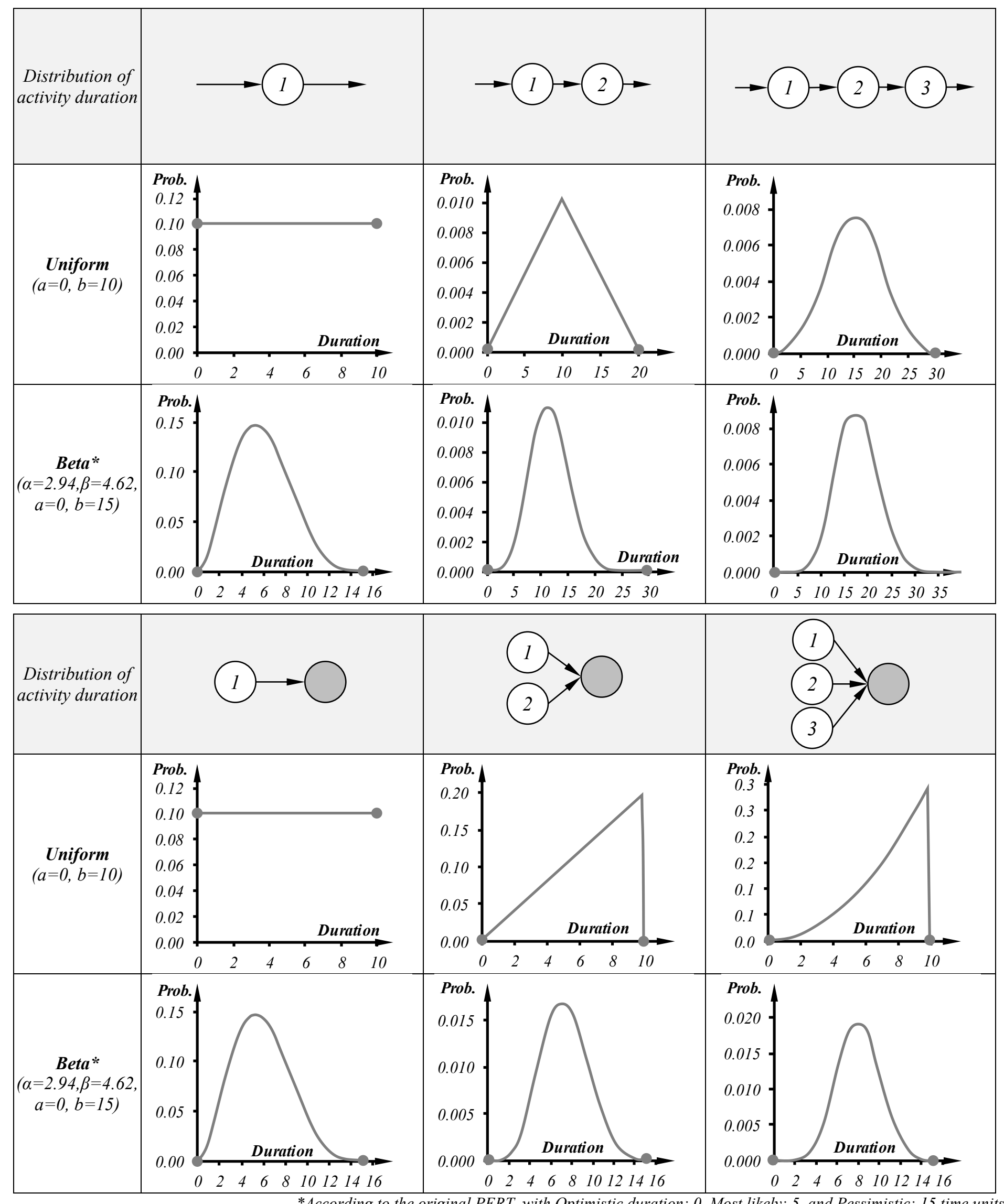

Fig. 1. Comparison of two probability distributions alteration as more activities are added 


\begin{tabular}{|c|c|c|}
\hline Precedence type & $\begin{array}{c}\text { Activity-on-Node }(\mathrm{AoN}) \\
\text { representation }\end{array}$ & $\begin{array}{c}\text { Finish-Start } \\
\text { equivalent }\end{array}$ \\
\hline \multicolumn{3}{|l|}{$\begin{array}{l}\text { Finishes } i, \text { Starts } j \\
\text { with a time lag } t \\
\quad(\text { omit if } t=0)\end{array}$} \\
\hline \multicolumn{3}{|l|}{$\begin{array}{l}\text { Starts } i, \text { Starts } j \\
\text { with a time lag } t\end{array}$} \\
\hline \multicolumn{3}{|l|}{$\begin{array}{c}\text { Finishes } i \text {, Finishes } j \\
\text { with a time lag } t\end{array}$} \\
\hline $\begin{array}{l}\text { Starts } i, \text { Finishes } j \\
\text { with a time lag } t\end{array}$ & & ( $j$ is not relevant) \\
\hline
\end{tabular}

Fig. 2. Array of activity precedences in M-PERT 


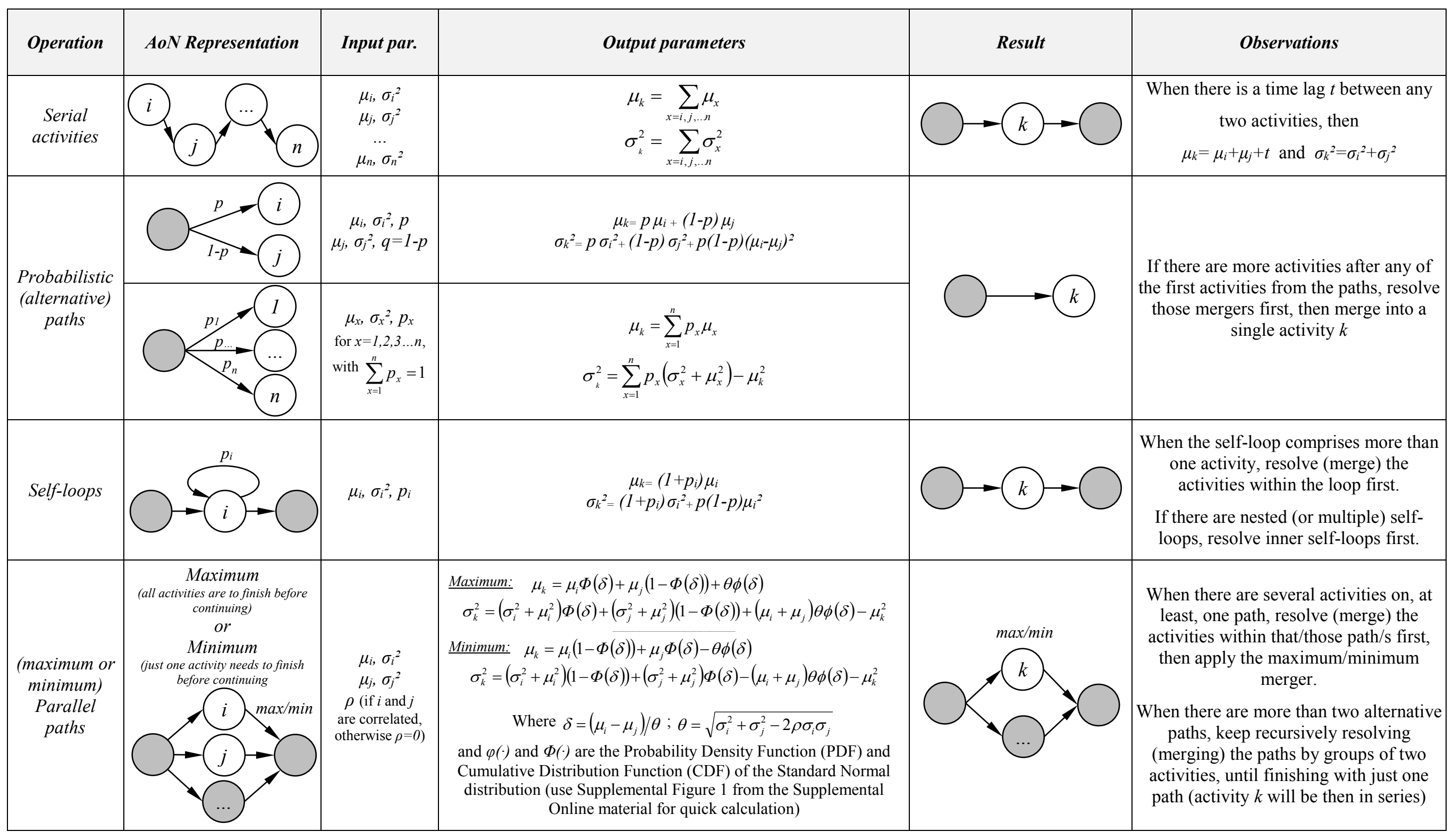

Fig. 3. Array of activity mergers in M-PERT 


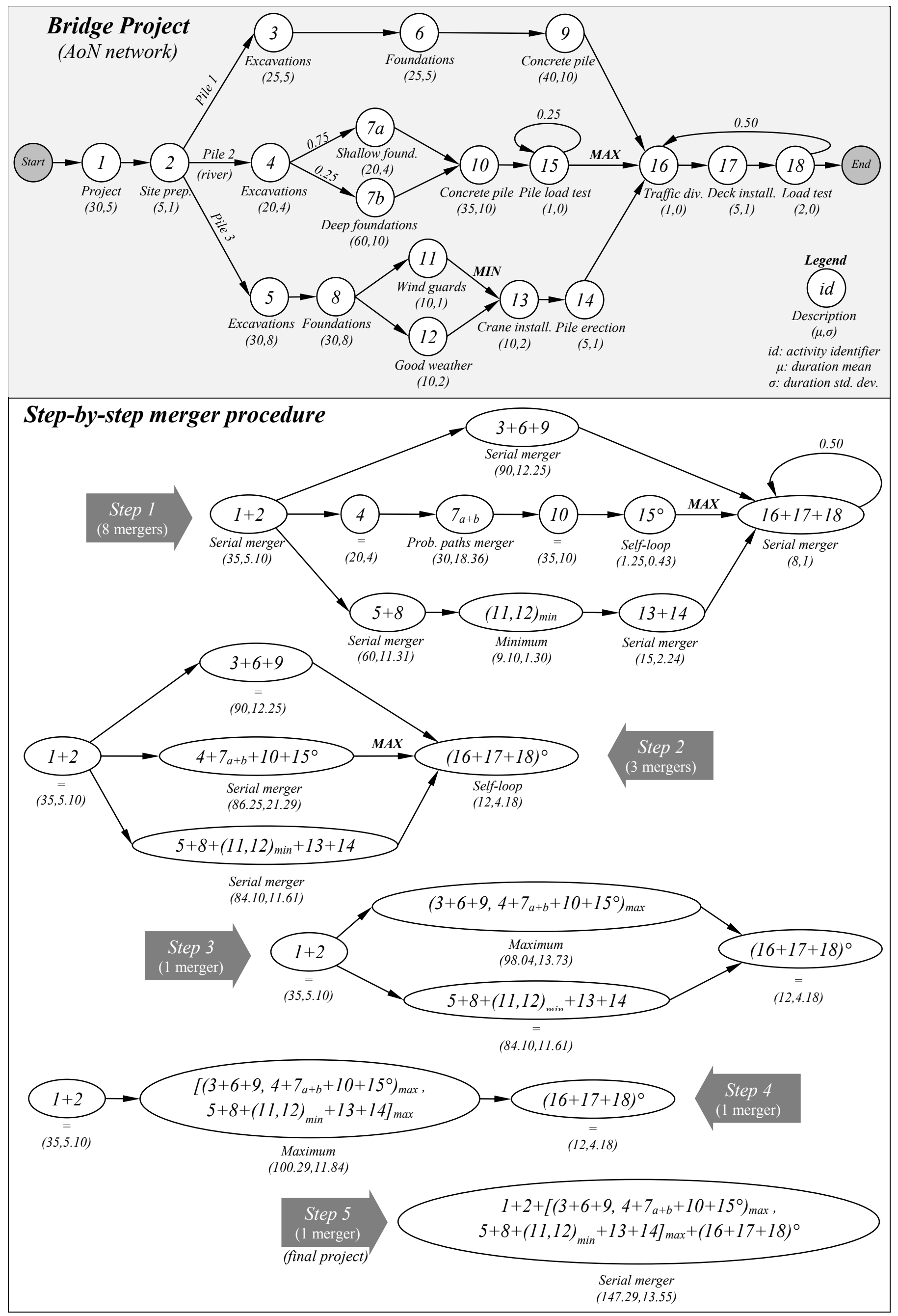

Fig. 4. M-PERT application in a fictitious simplified bridge project 


\begin{tabular}{|c|c|c|c|c|c|}
\hline Path & Activities & $\mu_{k}$ & $A$ & $B$ & $C$ \\
\hline$A$ & $1-2-4-7-9$ & $\begin{array}{c}\mu_{A}= \\
\mu_{1}+\mu_{2}+\mu_{4}+\mu_{7}+\mu_{9}\end{array}$ & $\begin{array}{c}\sigma_{A}^{2}= \\
\sigma_{1}^{2}+\sigma_{2}^{2}+\sigma_{4}^{2}+\sigma_{7}^{2}+\sigma_{9}^{2}\end{array}$ & $\begin{array}{c}C O V_{A B}= \\
\sigma_{1}^{2}+\sigma_{2}^{2}+\sigma_{9}^{2}\end{array}$ & $\begin{array}{l}C O V_{A C}= \\
\sigma_{1}^{2}+\sigma_{9}^{2}\end{array}$ \\
\hline$B$ & $1-2-5-8-9$ & $\begin{array}{c}\mu_{B}= \\
\mu_{1}+\mu_{2}+\mu_{5}+\mu_{8}+\mu_{9}\end{array}$ & $C O V_{B A}=C O V_{A B}$ & $\begin{array}{c}\sigma_{B}{ }= \\
\sigma^{2}+\sigma^{2}+\sigma^{2}+\sigma_{8}^{2}+\sigma_{9}^{2}\end{array}$ & $\begin{array}{c}C O V_{B C}= \\
\sigma^{2}+\sigma_{8}^{2}+\sigma_{9}^{2}\end{array}$ \\
\hline C & $1-3-6-8-9$ & $\begin{array}{c}\mu_{C}= \\
\mu_{1}+\mu_{3}+\mu_{\sigma}+\mu_{8}+\mu_{9}\end{array}$ & $C O V_{C A}=C O V_{A C}$ & $C O V_{C B}=C O V_{B C}$ & $\begin{array}{c}\sigma^{2}{ }^{=} \\
\sigma^{2}+\sigma^{2}{ }_{3}+\sigma^{2}{ }_{6}+\sigma_{8}^{2}+\sigma_{9}^{2}\end{array}$ \\
\hline & & & & & $\overbrace{\substack{\left(\mu_{i}, \sigma_{j}\right) \\
\text { i: activity identifier } \\
\mu_{i}: \text { : } u r a t i o n \\
\sigma_{i}: \text { duration std. dev. }}}^{\text {Legend }}$ \\
\hline Path & Activities & $\mu_{k}$ & $A$ & $B$ & $C$ \\
\hline$A$ & $1-3$ & $\mu_{A}=\mu_{1}+\mu_{3}$ & $\sigma_{A}^{2}=\sigma_{1}^{2}+\sigma_{3}^{2}$ & $C O V_{A B}=\sigma_{1}^{2}$ & $C O V_{A C}=\sigma_{3}^{2}$ \\
\hline$B$ & $1-4$ & $\mu_{B}=\mu_{I}+\mu_{4}$ & $C O V_{B A}=C O V_{A B}$ & $\sigma_{B}^{2}=\sigma_{1}^{2}+\sigma_{4}^{2}$ & $C O V_{B C}=0$ \\
\hline$C$ & $2-3$ & $\mu_{C}=\mu_{2}+\mu_{3}$ & $C O V_{C A}=C O V_{A C}$ & $C O V_{C B}=C O V_{B C}$ & $\sigma_{C}^{2}=\sigma_{2}^{2}+\sigma_{3}^{2}$ \\
\hline
\end{tabular}

Fig. 5. Two examples of AoN networks with correlation and the calculation of their covariance matrix 


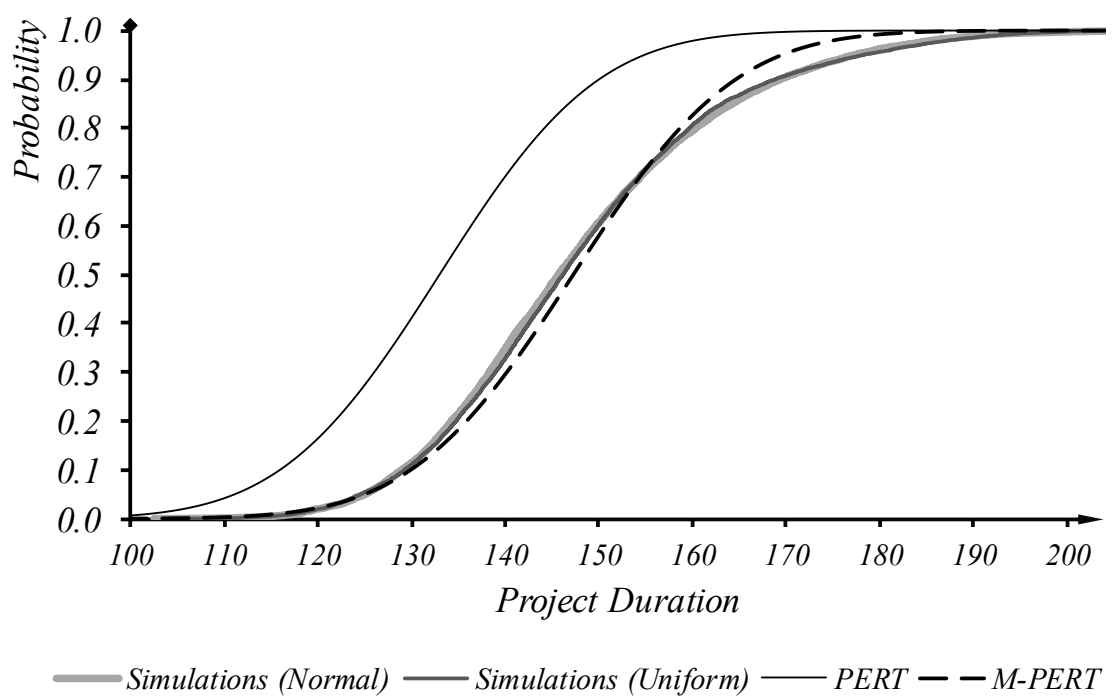

Fig. 6. Comparison of project duration estimation accuracy between PERT and M-PERT for the simplified bridge project 


\section{Supplemental data}

\section{Contents}

Detailed step-by-step calculations of the (Sub)network with correlation

Example Project 0 (page 2)

Alternative 1 (page 3)

Alternative 2 (general approach) (page 4)

Sample of 7 beginner-level exercises with increasing difficulty for learning M-PERT

Example Project 1 (page 7)

Example Project 2 (page 8)

Example Project 3 (page 9)

Example Project 4 (page 10)

Example Project 5 (page 12)

Example Project 6 (page 14)

Example Project 7 (page 16) 
Detailed step-by-step calculations for a (Sub)network with correlation.

\section{Example Project 0}
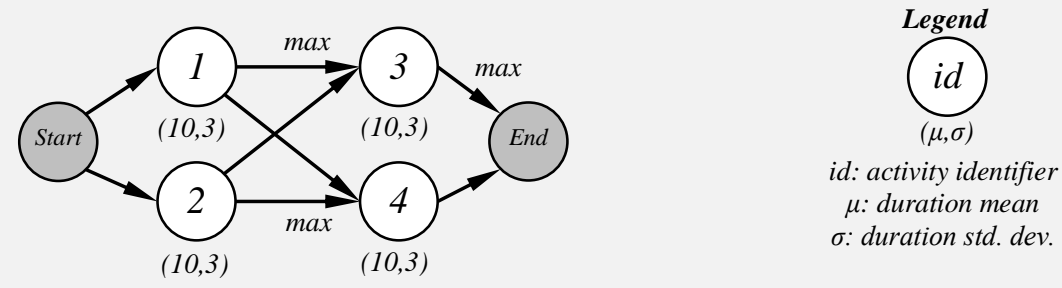

\section{Step-by-step merger procedure}

Alternative 1 (sometimes possible):

Step 1 (2 mergers)

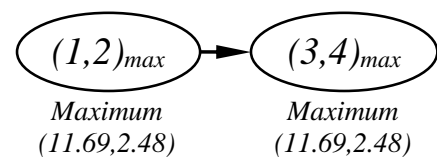

Alternative 2 (general procedure):

Step 1 (identify all posible paths)

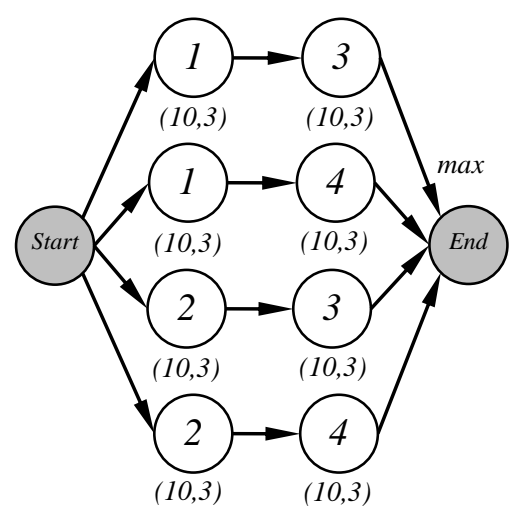

Step 2 (4 mergers)

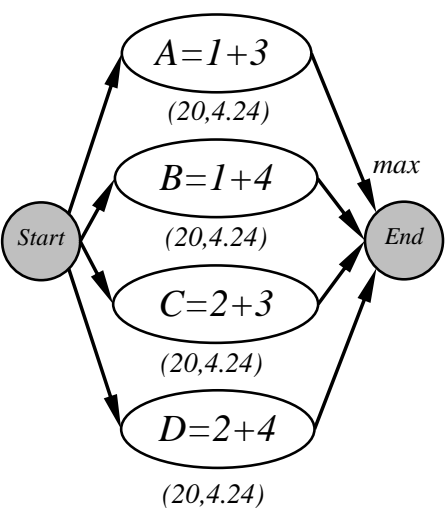

Step 2 (1 merger)

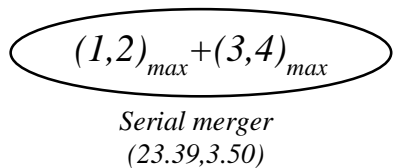

Step 3 (1 merger)

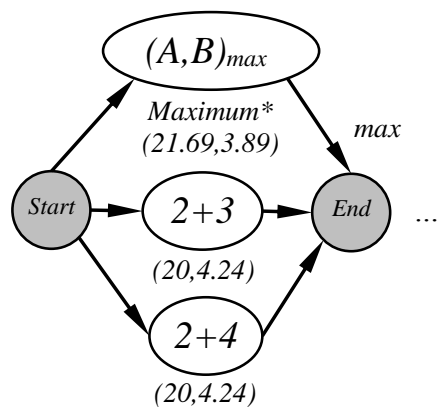

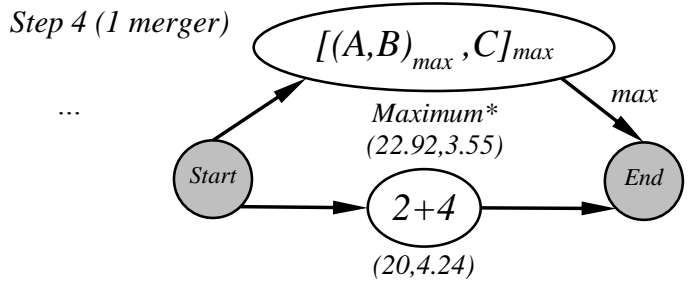

Step 5 (1 merger)

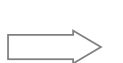

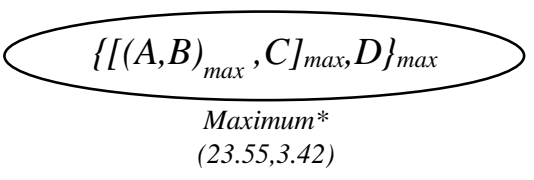

* merger with correlation between paths

\section{Results}

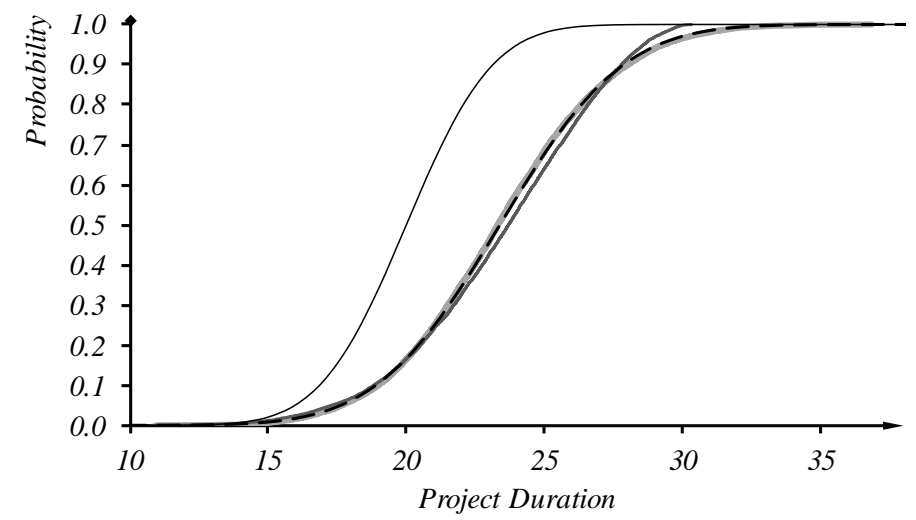

Simulations (Normal) $\longrightarrow$ Simulations (Uniform) $\longrightarrow$ PERT - - M-PERT 


\section{Alternative 1}

This alternative is possible due to the problem symmetry. If, for instance, the Finish-Start precedence relationship between nodes 2 and 4 had not existed, this alternative would have not been possible, only Alternative 2. However, when Alternative 1 is possible is always preferable since it is quicker and more accurate (since normally it entails fewer recursive applications of the maximum merger formulae)

\section{Step 1 (2 mergers)}

- Maximum merger between activities $i=1$ (with $\mu_{i}=10$ and $\sigma_{i}=3$ ) and $j=2$ (with $\mu_{j}=10$ and $\sigma_{j}=3$ ), then $k=(1,2)_{\max }$. Values of $\Phi(\cdot)$ and $\varphi(\cdot)$ can be calculated with the Supplemental Figure 1.

$$
\begin{aligned}
\theta & =\sqrt{\sigma_{i}^{2}+\sigma_{j}^{2}-2 \rho \sigma_{i} \sigma_{j}}=\sqrt{3^{2}+3^{2}-2 \cdot 0 \cdot 3 \cdot 3}=\sqrt{18}=4.243 \text { (there is no correlation between } 1 \text { and 2, i.e. } \rho=0 \text { ) } \\
\delta & =\left(\mu_{i}-\mu_{j}\right) / \theta=(10-10) / 4.243=0 \\
\mu_{k} & =\mu_{(1,2) \max }=\mu_{i} \Phi(\delta)+\mu_{j}(1-\Phi(\delta))+\theta \phi(\delta)=10 \Phi(0)+10(1-\Phi(0))+4.243 \phi(0)=10 \cdot 0.5+10 \cdot(1-0.5) \\
& +4.243 \cdot 0.399=11.693 \\
\sigma_{k}^{2} & =\sigma_{(1,2) \max }^{2}=\left(\sigma_{i}^{2}+\mu_{i}^{2}\right) \Phi(\delta)+\left(\sigma_{j}^{2}+\mu_{j}^{2}\right)(1-\Phi(\delta))+\left(\mu_{i}+\mu_{j}\right) \theta \phi(\delta)-\mu_{k}^{2}=\left(3^{2}+10^{2}\right) \Phi(0) \\
& +\left(3^{2}+10^{2}\right)(1-\Phi(0))+(10+10) 4.243 \phi(0)-11.693^{2}=109 \cdot 0.5+109 \cdot(1-0.5)+84.86 \cdot 0.399-136.726 \\
& =6.135 \rightarrow \sigma_{k}=\sqrt{6.135}=2.477
\end{aligned}
$$

- Maximum merger between activities $i=3$ (with $\mu_{i}=10$ and $\sigma_{i}=3$ ) and $j=4$ (with $\mu_{j}=10$ and $\sigma_{j}=3$ ), then $k=(3,4)_{\max }$.

In this case, it is obvious that since the means and variances of all the activities are the same, the result coincides with the maximum merger for $i=1$ and $j=2$. Therefore, $\mu_{k}=\mu_{(3,4) \max }=11.70$ and $\sigma_{k}=$ $\sigma_{(3,4) \max }=2.46$

\section{Step 2 (1 mergers)}

- Serial merger between activities $i=(1,2)_{\max }$. (with $\mu_{i}=11.693$ and $\sigma_{i}=2.477$ ) and $j=(3,4)_{\max }$ (with $\mu_{j}=11.693$ and $\left.\sigma_{j}=2.477\right)$, then $k=(1,2)_{\max }+(3,4)_{\max }$.

$$
\begin{aligned}
& \mu_{k}=\mu_{(1,2) \max +(3,4) \max }=\sum_{x=i, j, \ldots n} \mu_{x}=\mu_{i}+\mu_{j}=11.693+11.693=23.385 \rightarrow \text { Project Duration mean } \\
& \sigma_{k}^{2}=\sigma_{(1,2) \max +(3,4) \max }^{2}=\sum_{x=i, j, \ldots n} \sigma_{x}^{2}=\sigma_{i}^{2}+\sigma_{j}^{2}=2.477^{2}+2.477^{2}=12.270 \rightarrow \sigma_{k}=\sqrt{12.270}=3.503 \rightarrow \text { Project }
\end{aligned}
$$

\section{Duration standard deviation}

Now that $\mu_{p}=23.385$ and $\sigma_{p}=3.503$ are known, the probabilities of any possible project duration $(p)$ can be calculated by using the $\Phi(z)$ curve in Supplemental Figure 1 with $z=\left(D-\mu_{p}\right) / \sigma_{p}$. Or the other way around, that is, given a known probability $\Phi(z)=P$ (Y axis value), obtain the respective $z$ value and then $D=\mu_{p}+z \sigma_{p}$. 


\section{Alternative 2 (general approach)}

When alternative 1 option is not possible, a recursive (maximum) merger procedure for untangling the network is always possible between paths with shared activities. The only caution needed is to account for the correlation among the different paths when they are merged one by one until there is no one left to merge.

\section{Step 1 (identify all possible paths)}

- There are four possible paths in this (sub)network 1-3, 1-4, 2-3 and 2-4, which we will name for the sake of brevity for later calculations as $A, B, C$ and $D$, respectively.

It is also necessary to calculate all the activities means and variances, as well as their covariances. The covariance between any two paths is just the sum of the variances of those activities which are common between those two paths (unless another additional source of correlation is stated).

\begin{tabular}{|c|c|c|c|c|c|c|}
\hline Path $(i)$ & Activities & $\mu_{i}$ & $A$ & $B$ & $C$ & $D$ \\
\hline$A$ & 1,3 & $10+10=20$ & $3^{2}+3^{2}=18$ & $3^{2}=9$ & $3^{2}=9$ & 0 \\
$B$ & 1,4 & $10+10=20$ & $3^{2}=9$ & $3^{2}+3^{2}=18$ & 0 & $3^{2}=9$ \\
$C$ & 2,3 & $10+10=20$ & $3^{2}=9$ & 0 & $3^{2}+3^{2}=18$ & $3^{2}=9$ \\
$D$ & 2,4 & $10+10=20$ & 0 & $3^{2}=9$ & $3^{2}=9$ & $3^{2}+3^{2}=18$ \\
\hline
\end{tabular}

Values in the diagonal correspond to the variances, and those outside to the covariances between couples of paths. Finally, when the time comes later, the coefficient of correlation between two paths $i$ and $j$ will be calculated as $\rho=C O V_{i j} / \sigma_{i} \sigma_{j}$ where all the values are taken from the table above.

\section{Step 2 (4 mergers)}

- There are four serial mergers, as indicated in the table above. Detailed calculations are given for path $A$ applying the serial activities merger from Figure 3:

$$
\mu_{A}=\mu_{l}+\mu_{3}=20 \quad \sigma_{A}=\sqrt{\sigma_{1}^{2}+\sigma_{3}^{2}}=\sqrt{3^{2}+3^{2}}=\sqrt{18}=4.243
$$

Since the rest of the paths (in this example) have the same duration means and standard deviations, then: $\mu_{A}=\mu_{B}=\mu_{C}=\mu_{D}=20$ and $\sigma_{A}=\sigma_{B}=\sigma_{C}=\sigma_{D}=4.243$.

Step 3 (1 merger)

As a general rule all paths will be ordered from longest to shortest mean project duration and then merged. First, those two longest paths will be merged, and then one by one, in a recursive fashion, the rest will also be merged/added. This one-by-one order rule (unlike the quickest approach by couples of paths first, then by couples of couples of paths, and so on, when there is no correlation between paths) is necessary due to how the mathematical expressions of the correlation coefficients $\rho$ between maxima of paths are stated, since it is not possible (not easily at least) to calculate covariance between two different groups with multiple paths each.

- Maximum merger between paths $i=\mathrm{A}$ (with $\mu_{i}=20$ and $\sigma_{i}=4.243$ ) and $j=\mathrm{B}$ (with $\mu_{j}=20$ and $\sigma_{j}=4.243$ ), then $k=(A, B)_{\max }$. Values of $\Phi(\cdot)$ and $\varphi(\cdot)$ can be calculated with the Supplemental Figure 1.

$\rho_{A B}=\frac{C O V_{i j}}{\sigma_{i} \sigma_{j}}=\frac{C O V_{A B}}{\sigma_{A} \sigma_{B}}=\frac{9}{4.243 \cdot 4.243}=0.5$ 


$$
\begin{aligned}
& \theta_{A B}=\sqrt{\sigma_{i}^{2}+\sigma_{j}^{2}-2 \rho \sigma_{i} \sigma_{j}}=\sqrt{\sigma_{A}^{2}+\sigma_{B}^{2}-2 \rho_{A B} \sigma_{A} \sigma_{B}}=\sqrt{4.243^{2}+4.243^{2}-2 \cdot 0.5 \cdot 4.243 \cdot 4.243}=\sqrt{18}=4.243 \\
& \delta_{A B}=\left(\mu_{i}-\mu_{j}\right) / \theta=\left(\mu_{A}-\mu_{B}\right) / \theta_{A B}=(20-20) / 4.243=0 \\
& \mu_{k}=\mu_{(A, B) \max }=\mu_{i} \Phi(\delta)+\mu_{j}(1-\Phi(\delta))+\theta \phi(\delta)=\mu_{A} \Phi\left(\delta_{A B}\right)+\mu_{j}\left(1-\Phi\left(\delta_{A B}\right)\right)+\theta_{A B} \phi\left(\delta_{A B}\right)= \\
& \quad=20 \Phi(0)+20(1-\Phi(0))+4.243 \phi(0)=20 \cdot 0.5+20 \cdot(1-0.5)+4.243 \cdot 0.399=21.693 \rightarrow \mu_{A B} \\
& \sigma_{k}^{2}=\sigma_{(A, B) \max }^{2}=\left(\sigma_{i}^{2}+\mu_{i}^{2}\right) \Phi(\delta)+\left(\sigma_{j}^{2}+\mu_{j}^{2}\right)(1-\Phi(\delta))+\left(\mu_{i}+\mu_{j}\right) \theta \phi(\delta)-\mu_{k}^{2}= \\
& =\left(\sigma_{A}^{2}+\mu_{A}^{2}\right) \Phi\left(\delta_{A B}\right)+\left(\sigma_{B}^{2}+\mu_{B}^{2}\right)\left(1-\Phi\left(\delta_{A B}\right)\right)+\left(\mu_{A}+\mu_{B}\right) \theta_{A B} \phi\left(\delta_{A B}\right)-\mu_{A B}^{2}= \\
& =\left(4.243^{2}+20^{2}\right) \Phi(0)+\left(4.243^{2}+20^{2}\right)(1-\Phi(0))+(20+20) 4.243 \phi(0)-21.693^{2}= \\
& =418 \cdot 0.5+418 \cdot(1-0.5)+169.72 \cdot 0.399-470.586=15.132 \rightarrow \sigma_{k}=\sqrt{15.132}=3.890 \rightarrow \sigma_{A B}
\end{aligned}
$$

Note that, for ease of notation, both results have been renamed as $\mu_{A B}$ and $\sigma_{A B}$.

\section{Step 4 (1 merger)}

- Maximum merger between paths $i=(A, B)_{\max }=\mathrm{AB}$ (with $\mu_{i}=21.693$ and $\sigma_{i}=3.890$ ) and $j=\mathrm{C}$ (with $\mu_{j}=20$ and $\left.\sigma_{j}=4.243\right)$, then $k=\left[(A, B)_{\max }, C\right]=A B C$. The expression for the coefficient of correlation calculation is derived from Sculli \& Shum (1991):

$$
\begin{aligned}
& \rho_{A B C}=\frac{\operatorname{COV}_{A C} \Phi\left(\delta_{A B}\right)+C O V_{B C}\left(1-\Phi\left(\delta_{A B}\right)\right)}{\sigma_{A B} \sigma_{C}}=\frac{9 \Phi(0)+0(1-\Phi(0))}{3.890 \cdot 4.243}=\frac{9 \cdot 0.5+0}{16.505}=0.273 \\
& \theta_{A B C}=\sqrt{\sigma_{A B}^{2}+\sigma_{C}^{2}-2 \rho_{A B C} \sigma_{A B} \sigma_{C}}=\sqrt{3.890^{2}+4.243^{2}-2 \cdot 0.273 \cdot 3.890 \cdot 4.243}=\sqrt{24.120}=4.913 \\
& \delta_{A B C}=\left(\mu_{A B}-\mu_{C}\right) / \theta_{A B C}=(21.693-20) / 4.913=0.345 \\
& \mu_{A B C}=\mu_{A B} \Phi\left(\delta_{A B C}\right)+\mu_{C}\left(1-\Phi\left(\delta_{A B C}\right)\right)+\theta_{A B C} \phi\left(\delta_{A B C}\right)=21.693 \Phi(0.345)+20(1-\Phi(0.345)) \\
& \quad+4.912 \phi(0.345)=21.693 \cdot 0.635+20 \cdot(1-0.635)+4.913 \cdot 0.376=22.921 \rightarrow \mu_{A B C} \\
& \sigma_{A B C}^{2}=\left(\sigma_{A B}^{2}+\mu_{A B}^{2}\right) \Phi\left(\delta_{A B C}\right)+\left(\sigma_{C}^{2}+\mu_{C}^{2}\right)\left(1-\Phi\left(\delta_{A B C}\right)\right)+\left(\mu_{A B}+\mu_{C}\right) \theta_{A B C} \phi\left(\delta_{A B C}\right)-\mu_{A B C}^{2}= \\
& \quad=\left(3.890^{2}+21.693^{2}\right) \Phi(0.345)+\left(4.243^{2}+20^{2}\right)(1-\Phi(0.345))+(21.693+20) 4.913 \phi(0.345) \\
& \quad-22.921^{2}=485.718 \cdot 0.635+418 \cdot(1-0.635)+204.754 \cdot 0.376-525.372=12.617 \rightarrow \\
& \sigma_{k}=\sqrt{12.617}=3.548 \rightarrow \sigma_{A B C}
\end{aligned}
$$

\section{Step 5 (1 merger)}

- Maximum merger between paths $i=\left[(A, B)_{\max }, C\right]_{\max }=\mathrm{ABC}$ (with $\mu_{i}=22.921$ and $\sigma_{i}=3.548$ ) and $j=\mathrm{D}$ (with $\mu_{j}=20$ and $\sigma_{j}=4.243$ ), then $k=\left\{\left[(A, B)_{\max }, C\right]_{\max }, D\right\}_{\max }=A B C D$. The expression for the coefficient of correlation calculation is derived from Sculli \& Shum (1991):

$$
\begin{aligned}
& \rho_{A B C D}=\frac{\left[C O V_{A D} \Phi\left(\delta_{A B}\right)+C O V_{B D}\left(1-\Phi\left(\delta_{A B}\right)\right)\right] \Phi\left(\delta_{A B C}\right)+C O V_{C D}\left(1-\Phi\left(\delta_{A B C}\right)\right)}{\sigma_{A B C} \sigma_{D}}= \\
& =\frac{[0 \Phi(0)+9(1-\Phi(0))] \Phi(0.345)+9(1-\Phi(0.345))}{3.548 \cdot 4.243}=\frac{9 \cdot(1-0.5) 0.635+9 \cdot(1-0.635)}{15.054}=0.408 \\
& \theta_{A B C D}=\sqrt{\sigma_{A B C}^{2}+\sigma_{D}^{2}-2 \rho_{A B C D} \sigma_{A B C} \sigma_{D}}=\sqrt{3.548^{2}+4.243^{2}-2 \cdot 0.408 \cdot 3.548 \cdot 4.243}=\sqrt{18.305}=4.278 \\
& \delta_{A B C D}=\left(\mu_{A B C}-\mu_{D}\right) / \theta_{A B C D}=(22.921-20) / 4.278=0.683
\end{aligned}
$$




$$
\begin{gathered}
\mu_{A B C D}=\mu_{A B C} \Phi\left(\delta_{A B C D}\right)+\mu_{D}\left(1-\Phi\left(\delta_{A B C D}\right)\right)+\theta_{A B C D} \phi\left(\delta_{A B C D}\right)=22.921 \Phi(0.683)+20(1-\Phi(0.683)) \\
\quad+4.278 \phi(0.683)=22.921 \cdot 0.753+20 \cdot(1-0.753)+4.278 \cdot 0.316=23.551 \rightarrow \text { Project Duration mean } \\
\sigma_{A B C D}^{2}=\left(\sigma_{A B C}^{2}+\mu_{A B C}^{2}\right) \Phi\left(\delta_{A B C D}\right)+\left(\sigma_{D}^{2}+\mu_{D}^{2}\right)\left(1-\Phi\left(\delta_{A B C D}\right)\right)+\left(\mu_{A B C}+\mu_{D}\right) \theta_{A B C D} \phi\left(\delta_{A B C D}\right)-\mu_{A B C D}^{2}= \\
\quad=\left(3.548^{2}+22.921^{2}\right) \Phi(0.683)+\left(4.243^{2}+20^{2}\right)(1-\Phi(0.683))+(22.921+20) 4.278 \phi(0.683) \\
\quad-23.551^{2}=537.960 \cdot 0.753+418 \cdot(1-0.753)+183.616 \cdot 0.316-554.650=11.695 \\
\rightarrow \sigma_{k}=\sqrt{11.695}=3.420 \rightarrow \text { Project Duration standard deviation }
\end{gathered}
$$

It is easy to see that, due to the recursive application of the maximum merger for the four paths, these results $\left(\mu_{p}=23.551\right.$ and $\left.\sigma_{p}=3.420\right)$ do not exactly coincide with the previous from alternative 1 $\left(\mu_{p}=23.385\right.$ and $\left.\sigma_{p}=3.503\right)$, but they are very close. Again, the probabilities of any possible project duration $(p)$ can be calculated exactly as was explained for the Alternative 1.

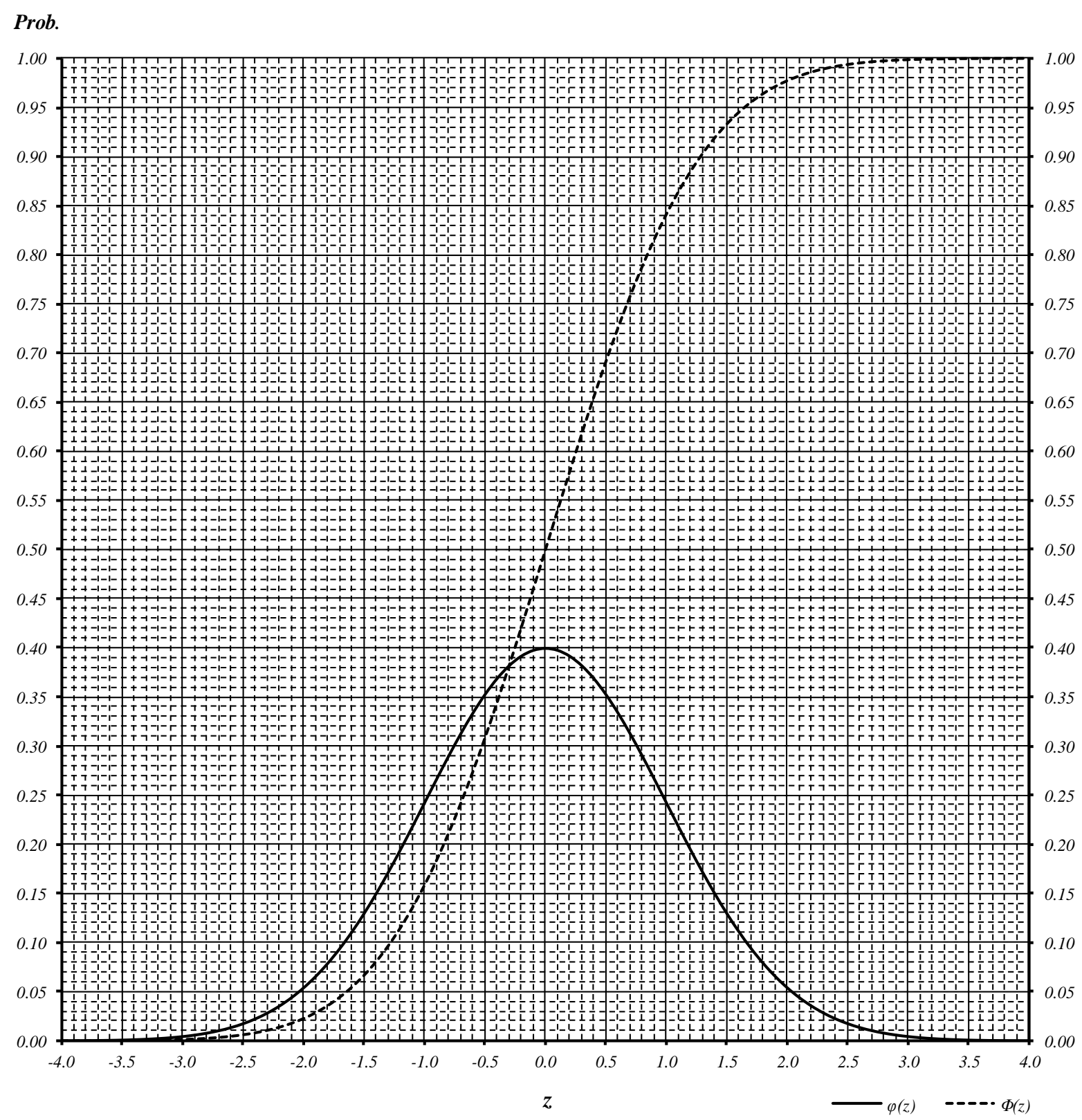

Supplemental Figure 1. Abacus for calculating the probability density function $\varphi(z)$ and the cumulative distribution function $\Phi(z)$ of the Standard Normal distribution 
Sample of 7 exercises (project schedules) of increasing difficulty for learning M-PERT

Note: for the calculations of the original PERT technique, self-loops are ignored (as they cannot be calculated with PERT) and it has been assumed that the Critical Path goes through the path with higher probabilities of happening in presence of probabilistic paths.

\section{Example Project 1}
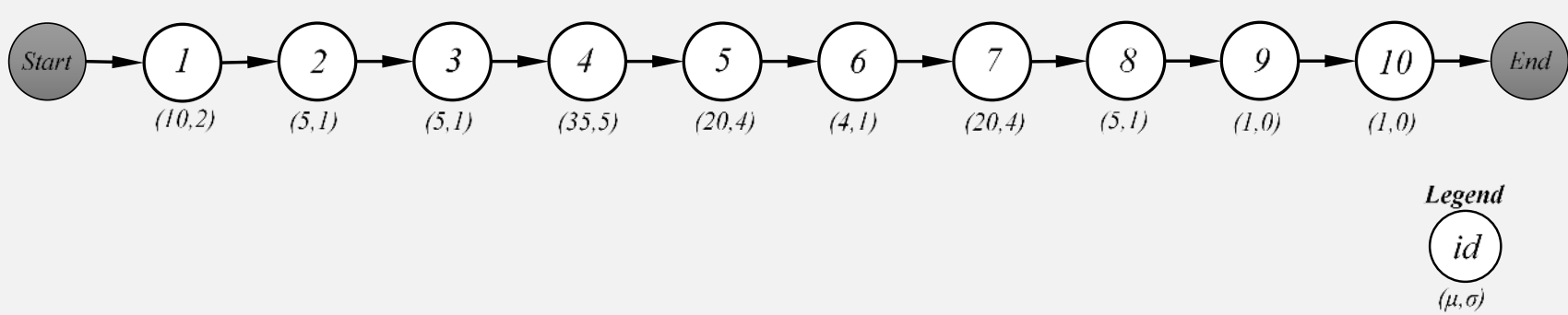

id: activity identifier

$\mu$ : duration mean

$\sigma$ : duration std. dev.

\section{Step-by-step merger procedure}
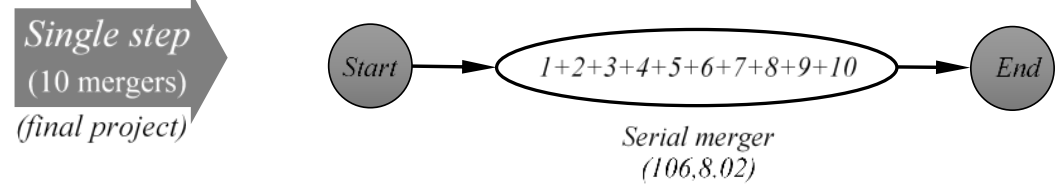

\section{Results}

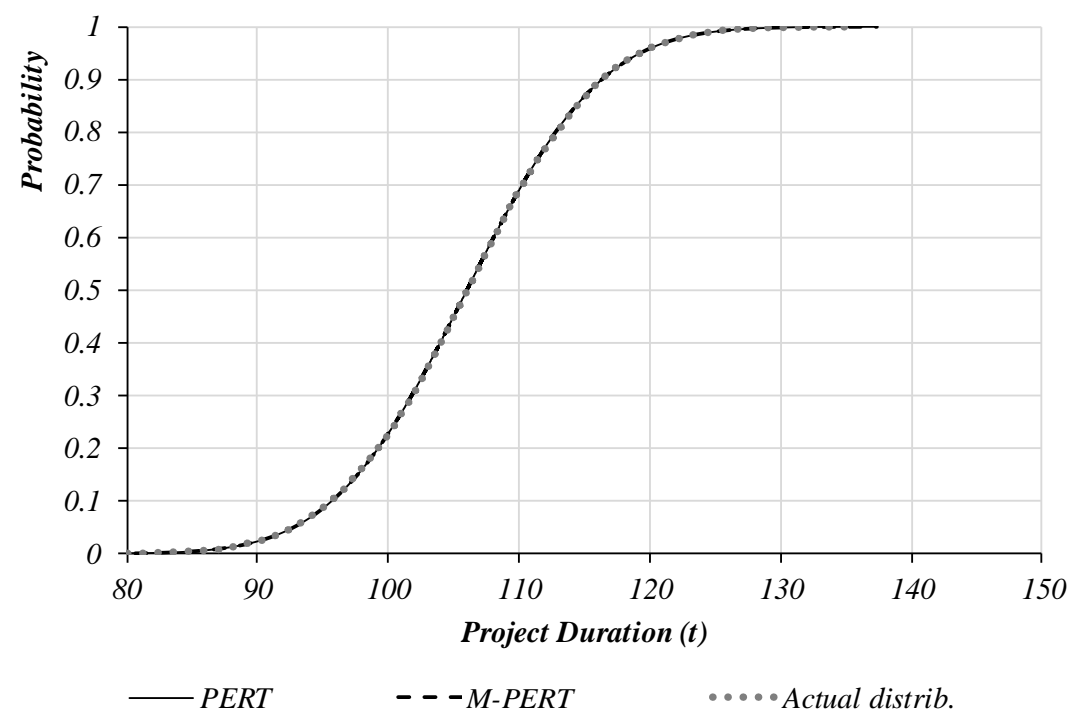




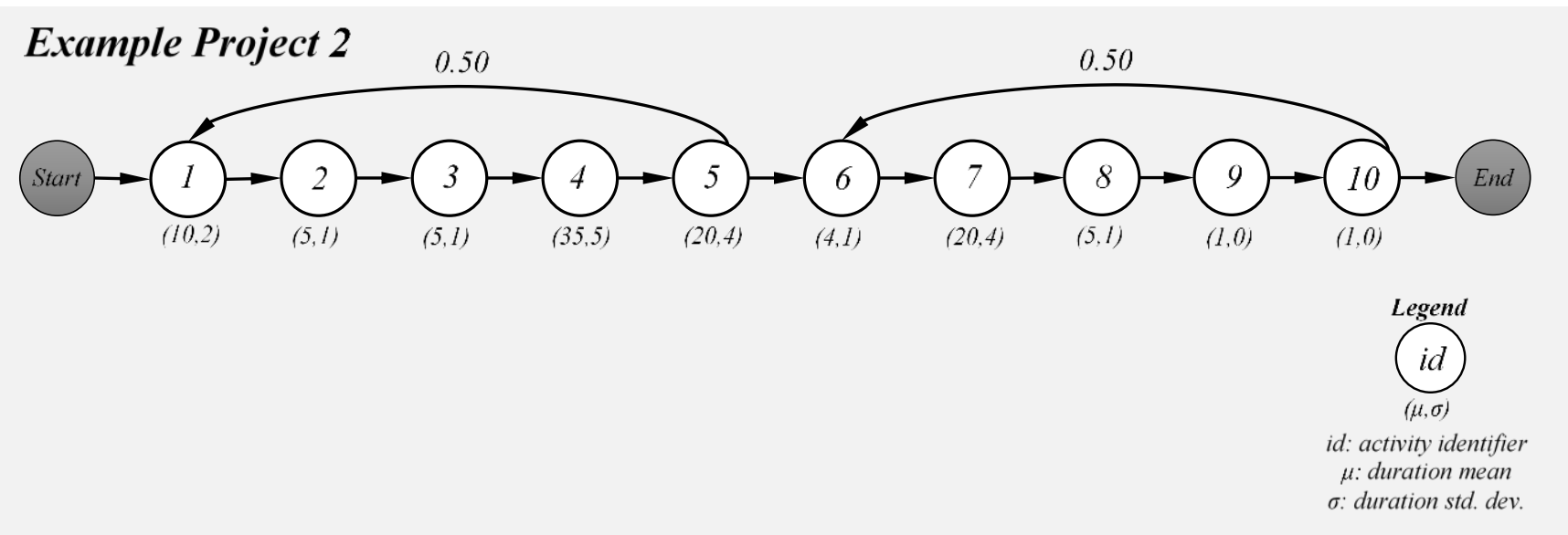

\section{Step-by-step merger procedure}
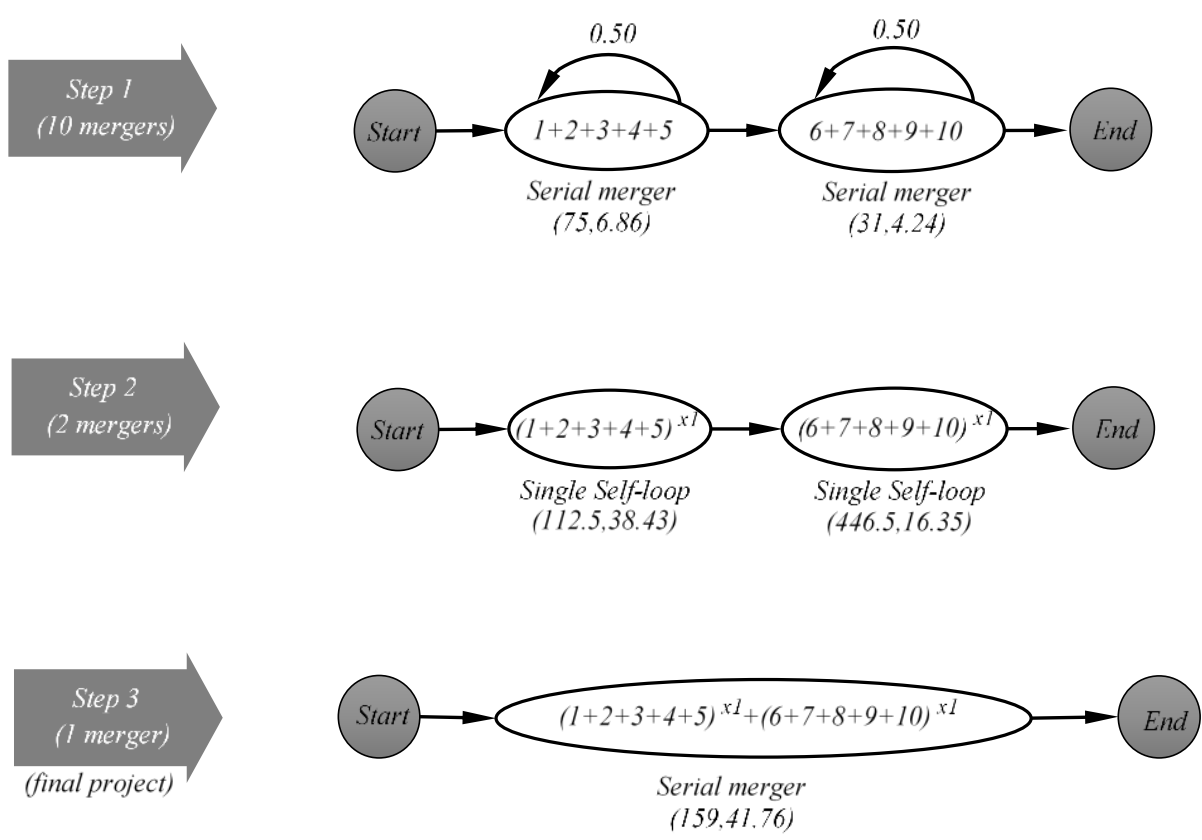

\section{Results}

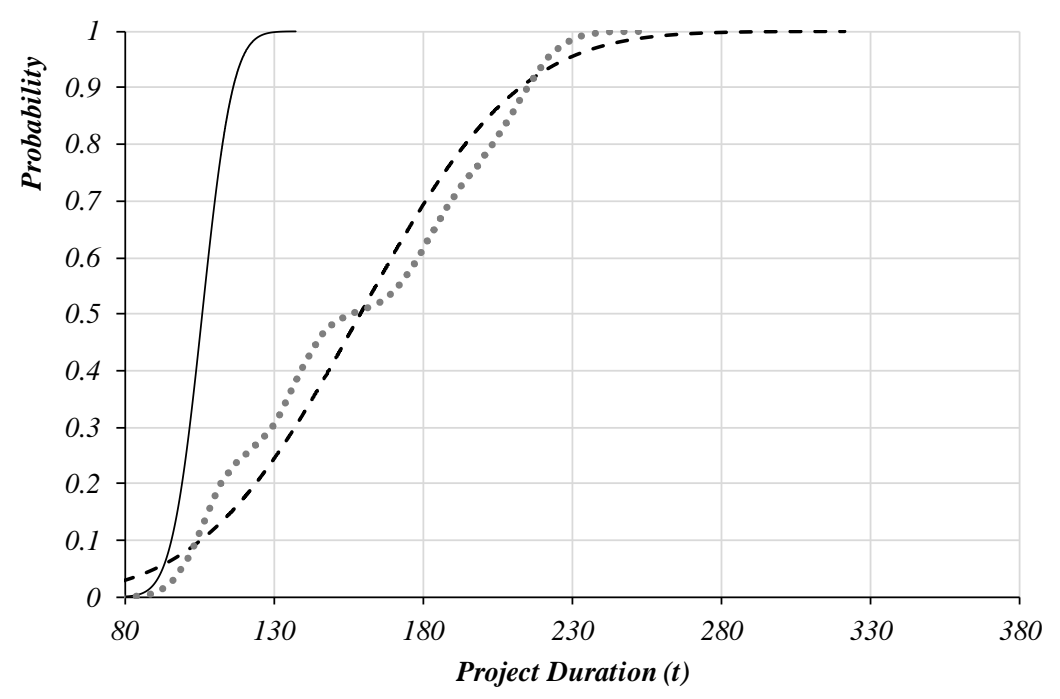

- PERT $\quad--M$-PERT $\quad \cdots$ Actual distrib. 


\section{Example Project 3}

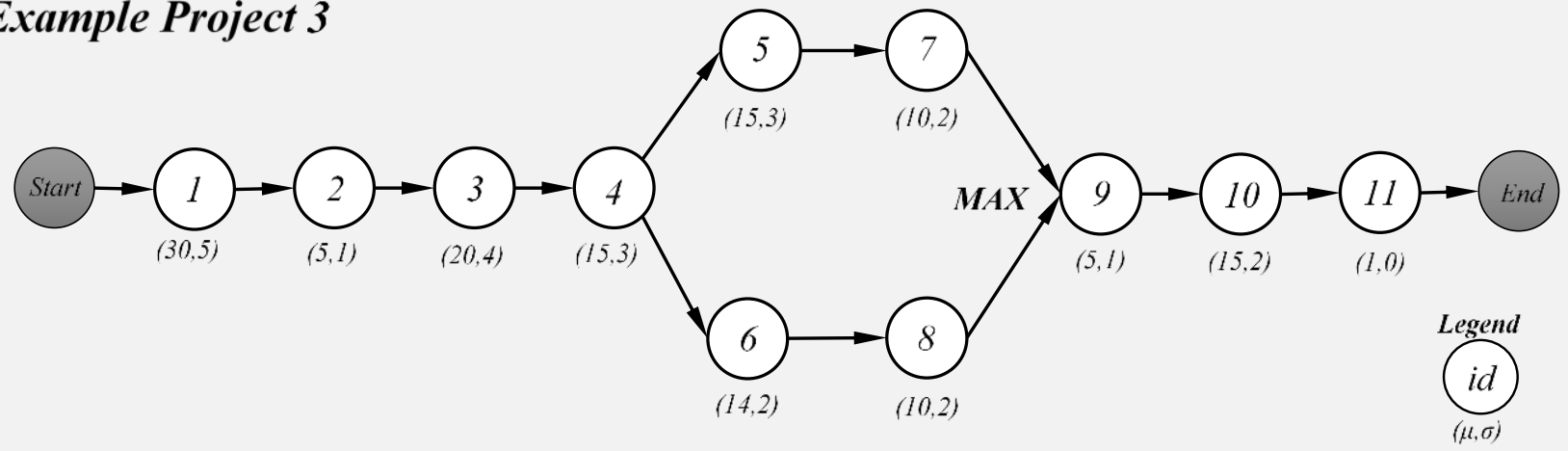

id: activity identifier

\section{Step-by-step merger procedure}
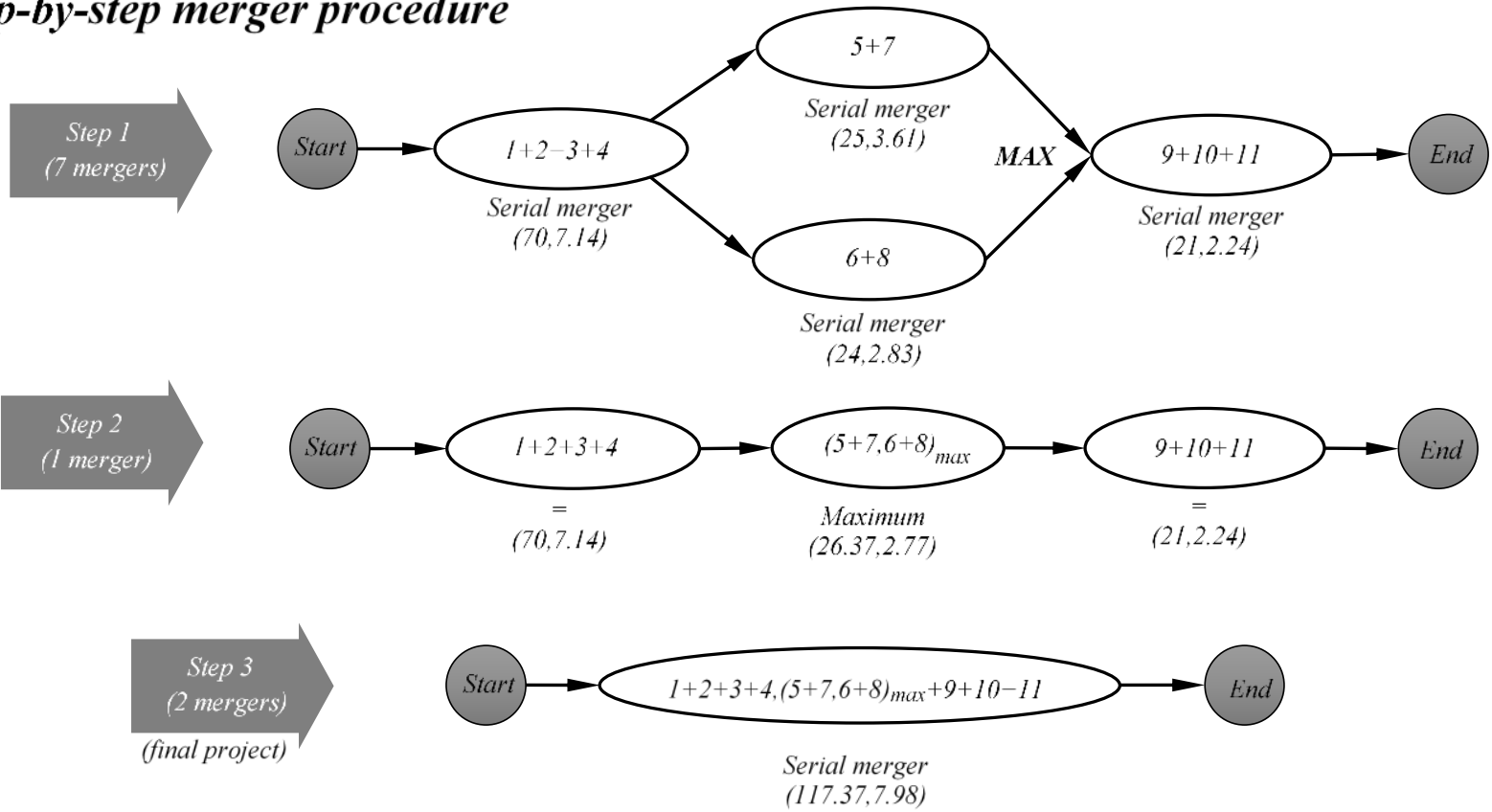

\section{Results}

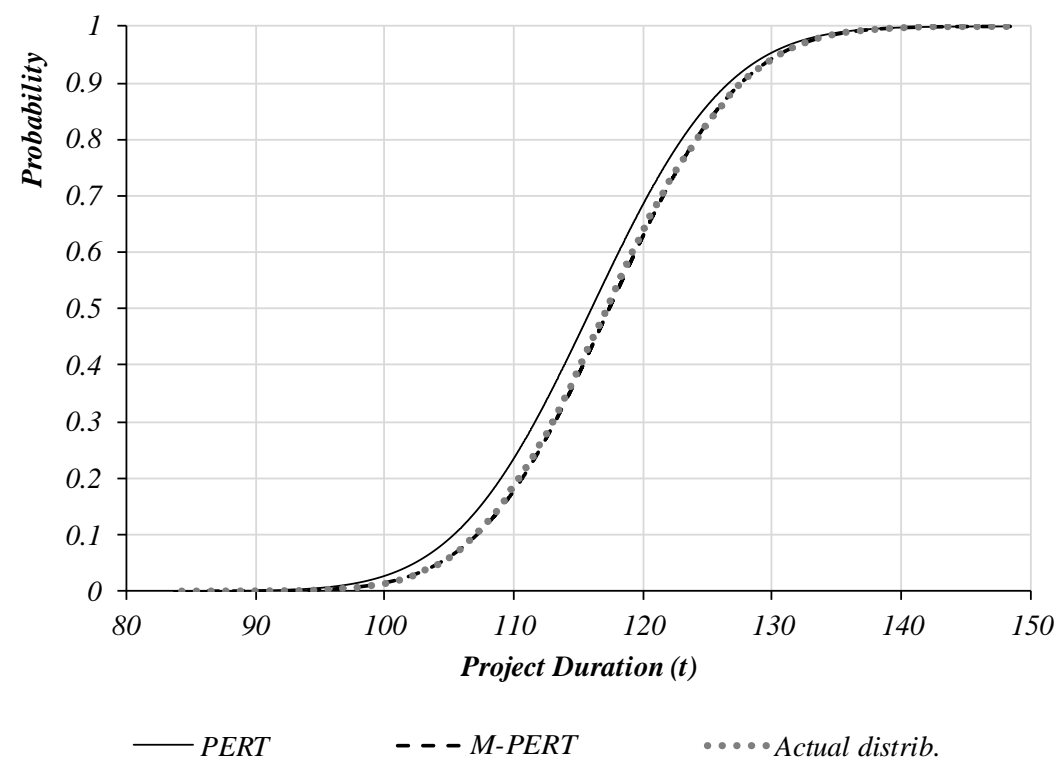




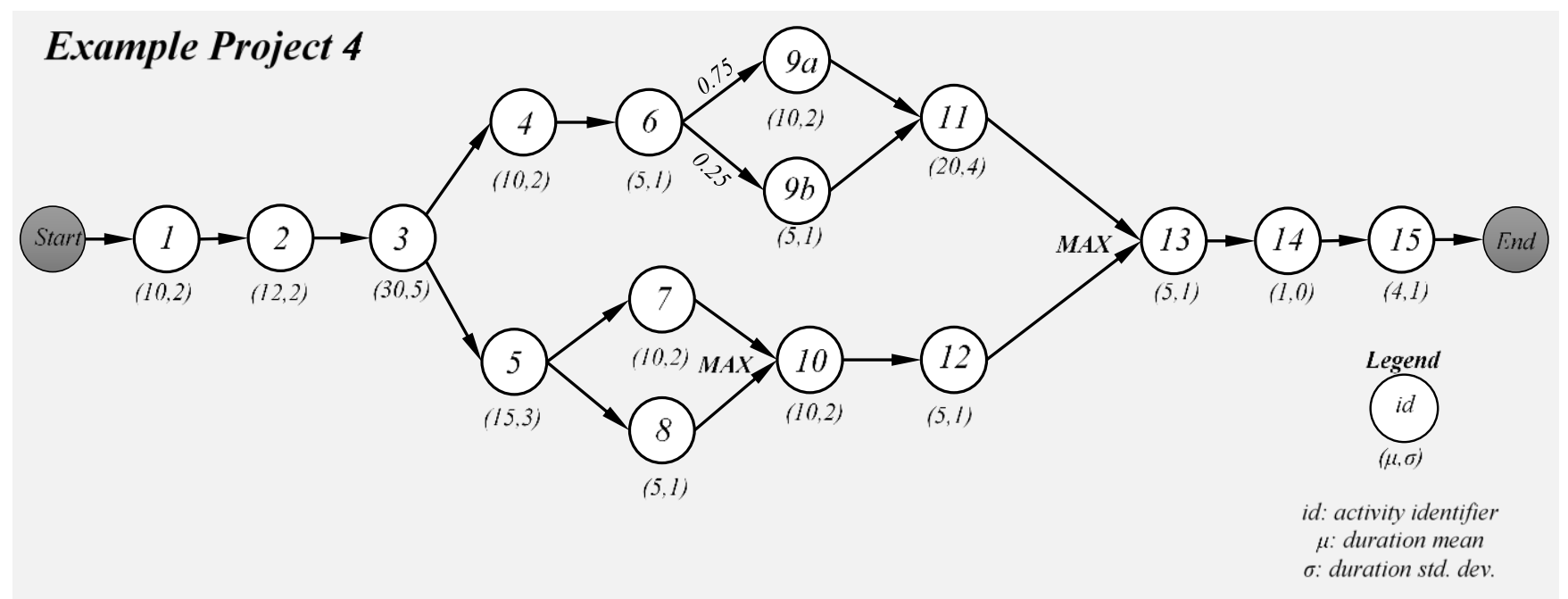

\section{Step-by-step merger procedure}
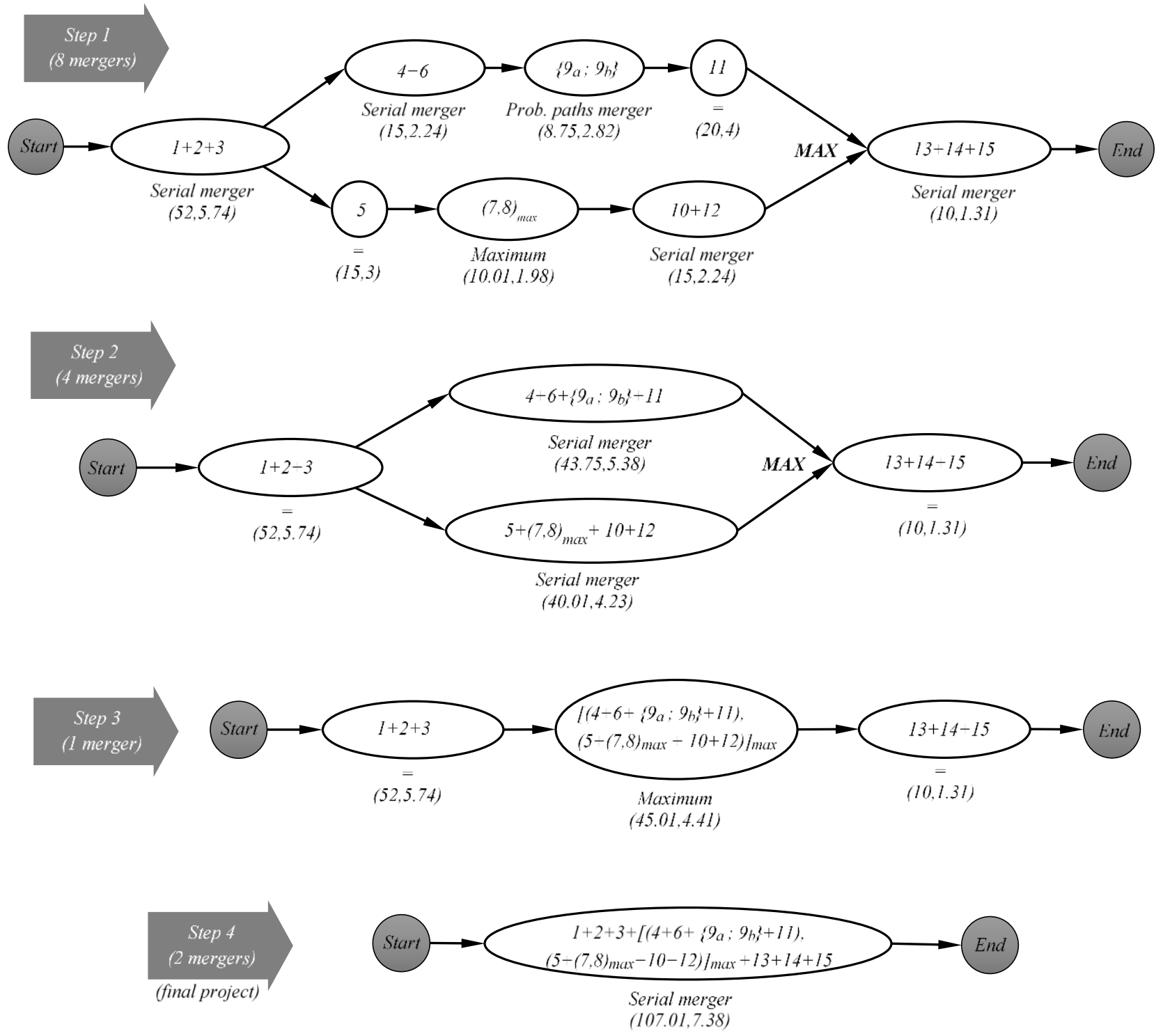


\section{Results}

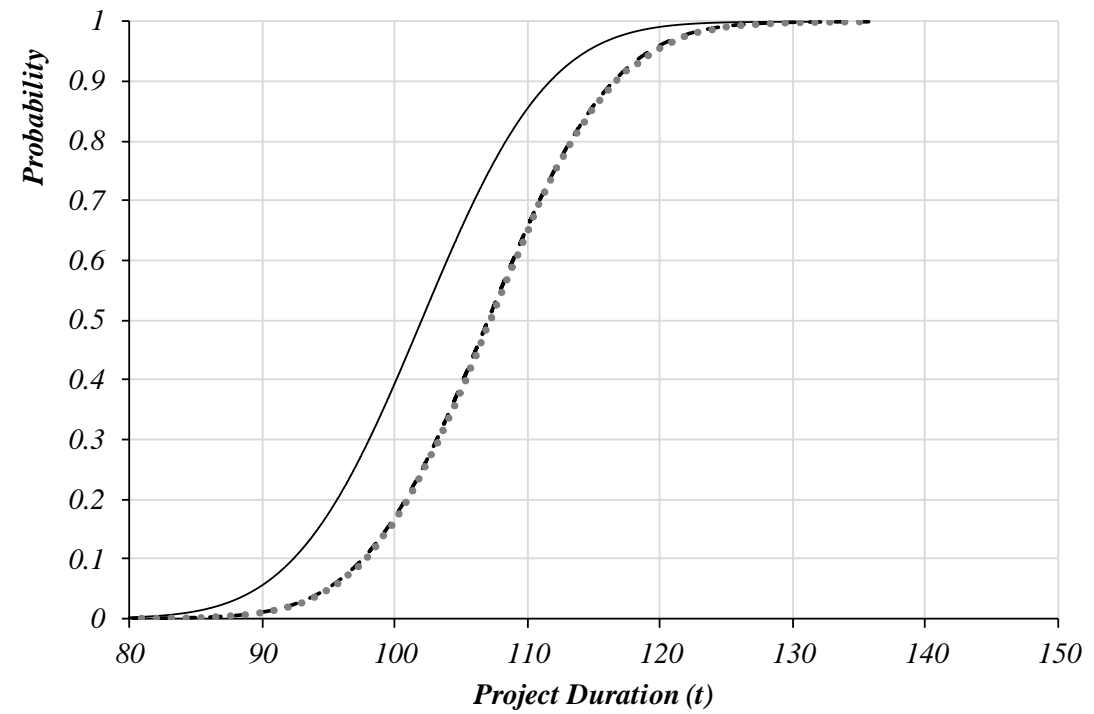

- PERT

- -M-PERT

.... Actual distrib 


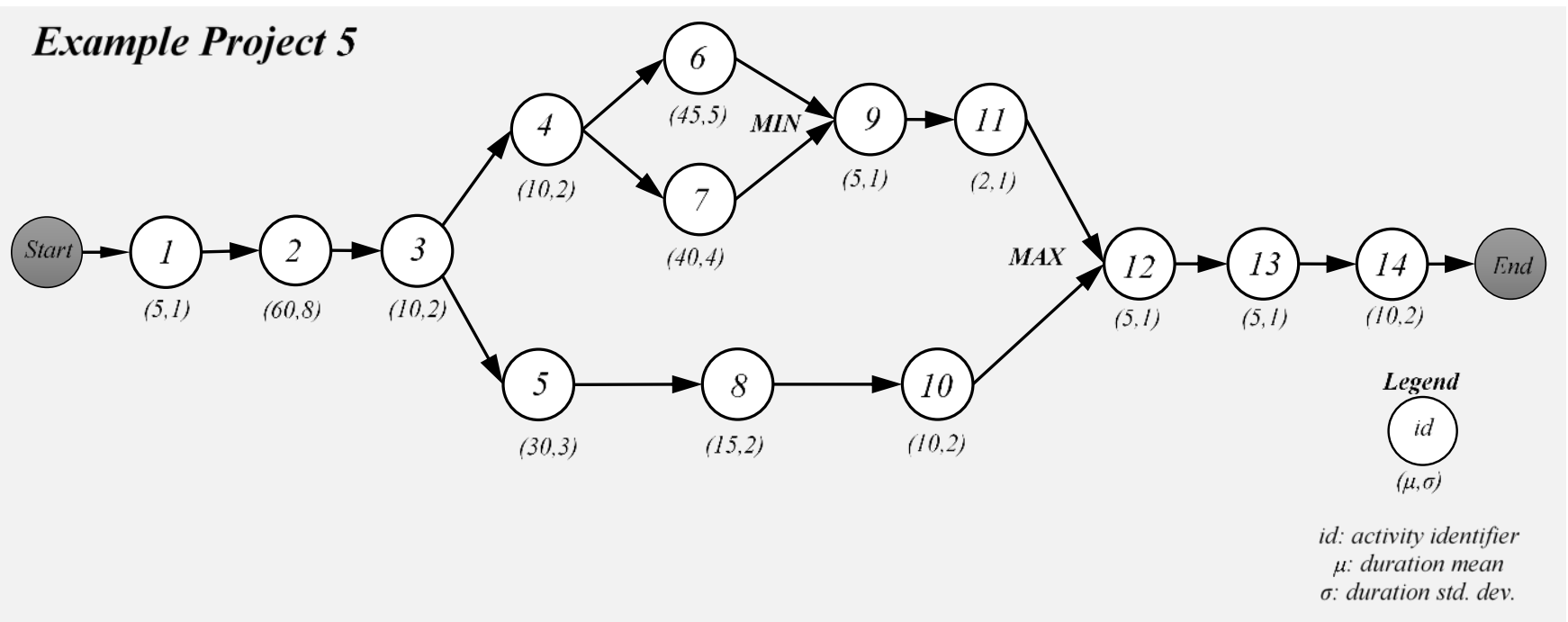

\section{Step-by-step merger procedure}
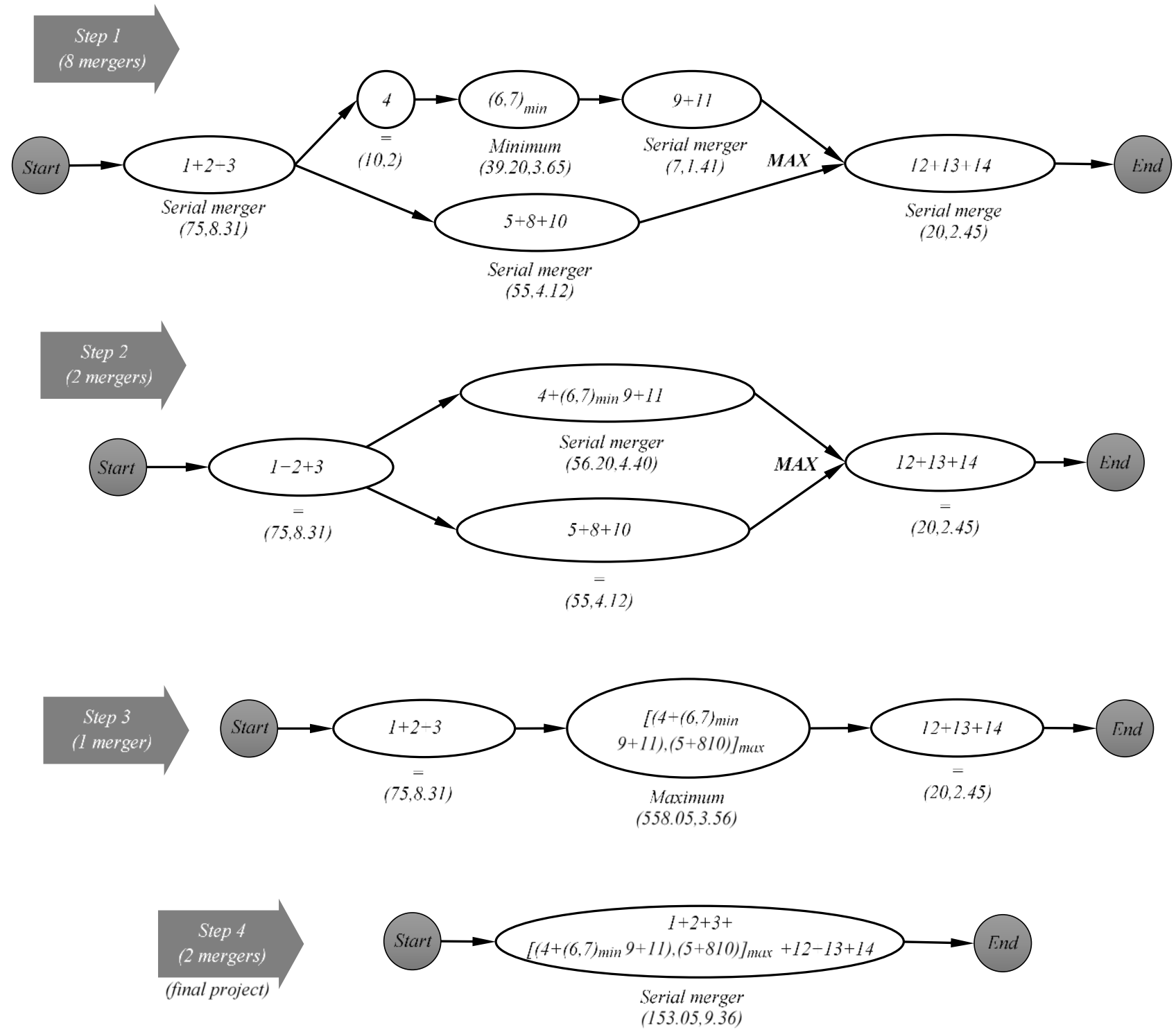
Results

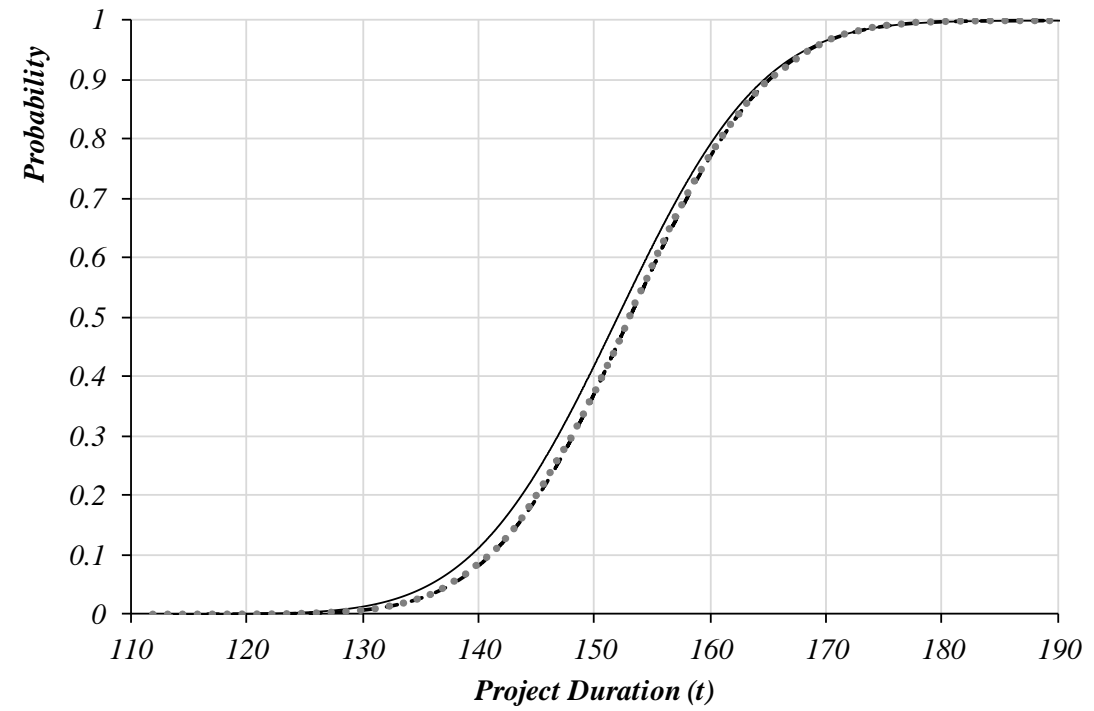

- PERT $\quad--M-P E R T \quad \cdots \cdots$ Actual distrib 


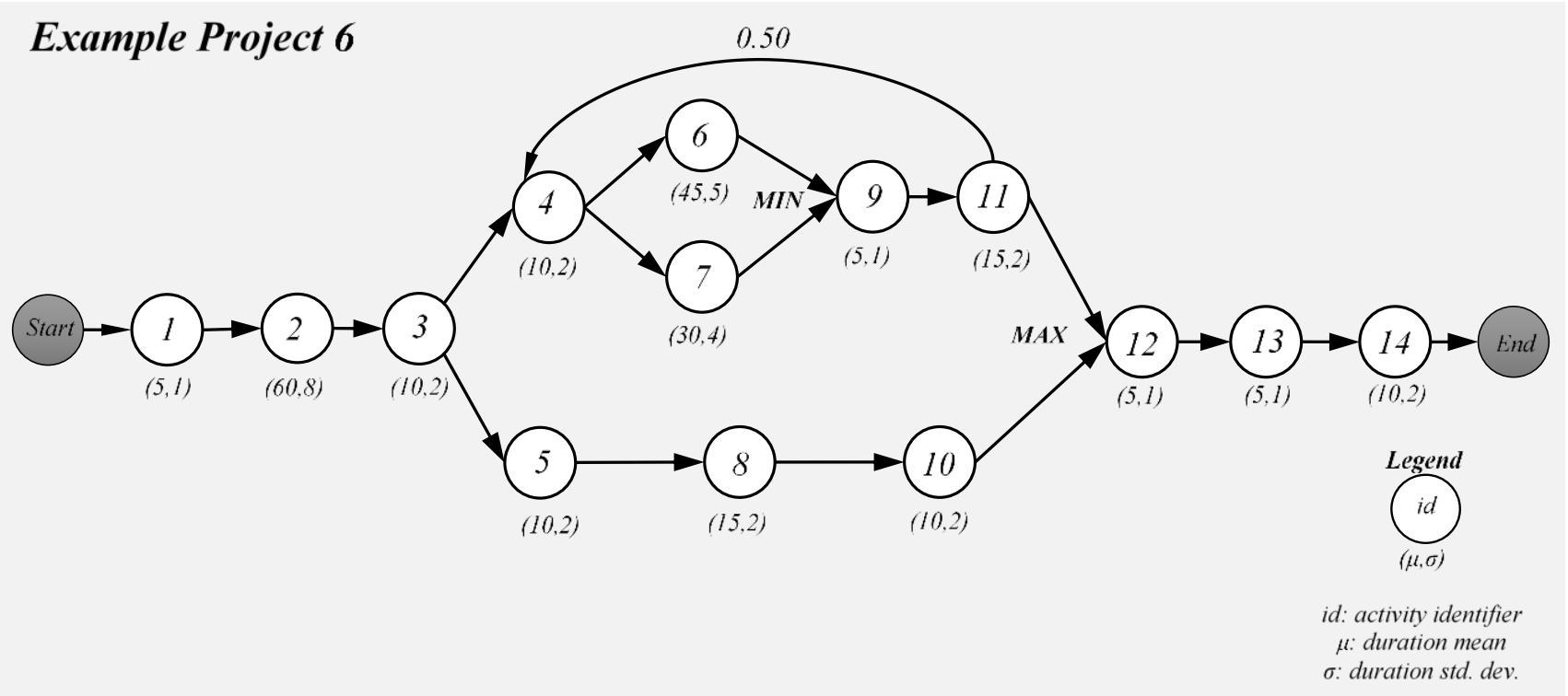

\section{Step-by-step merger procedure}
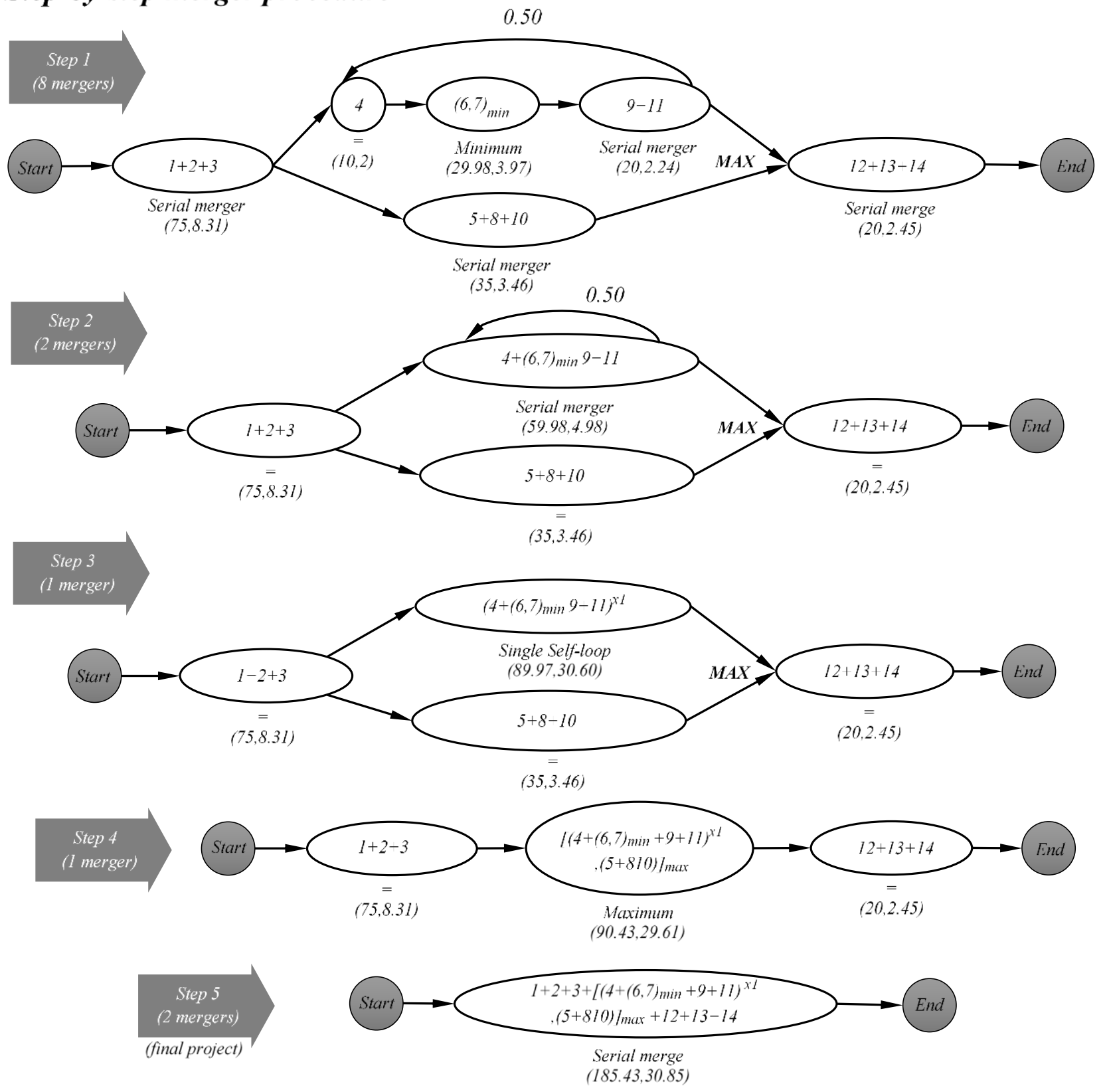


\section{Results}

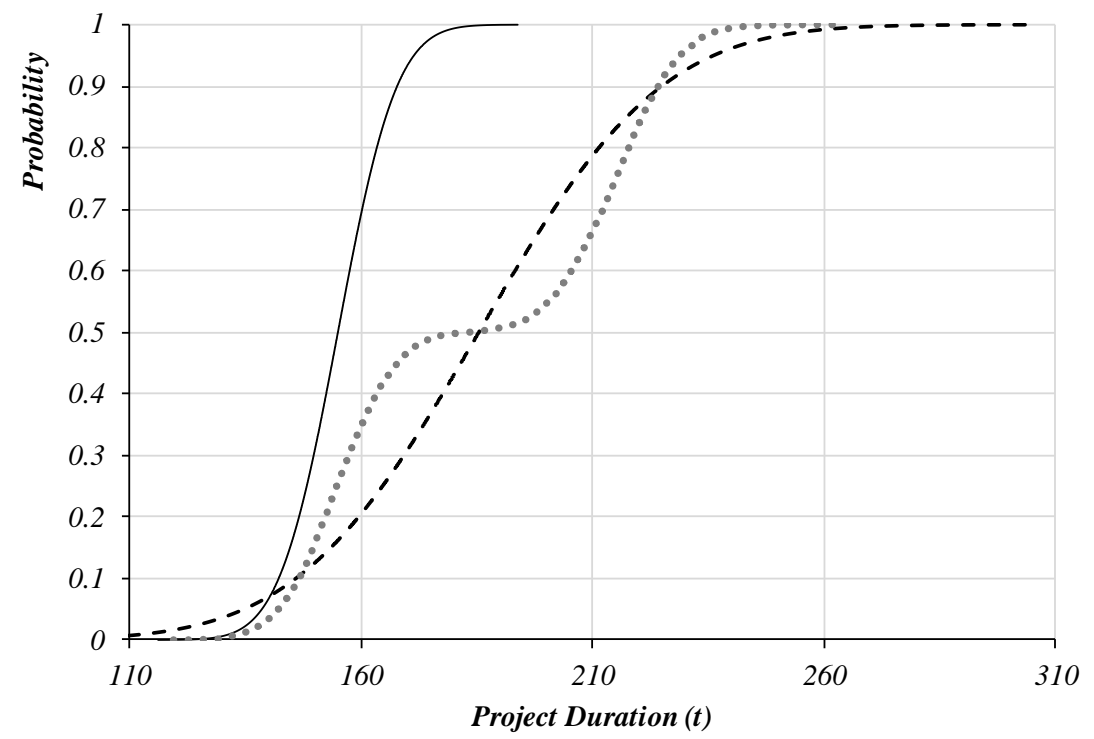

- PERT $\quad--M$-PERT $\cdots$ Actual distrib. 


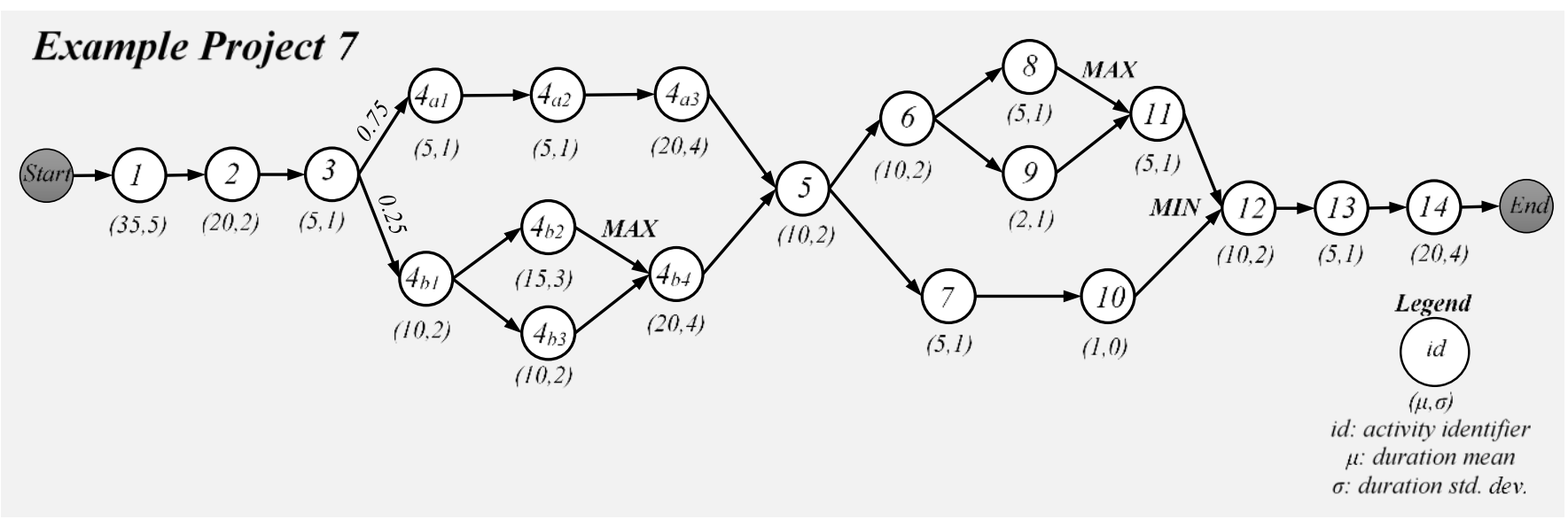

Step-by-step merger procedure
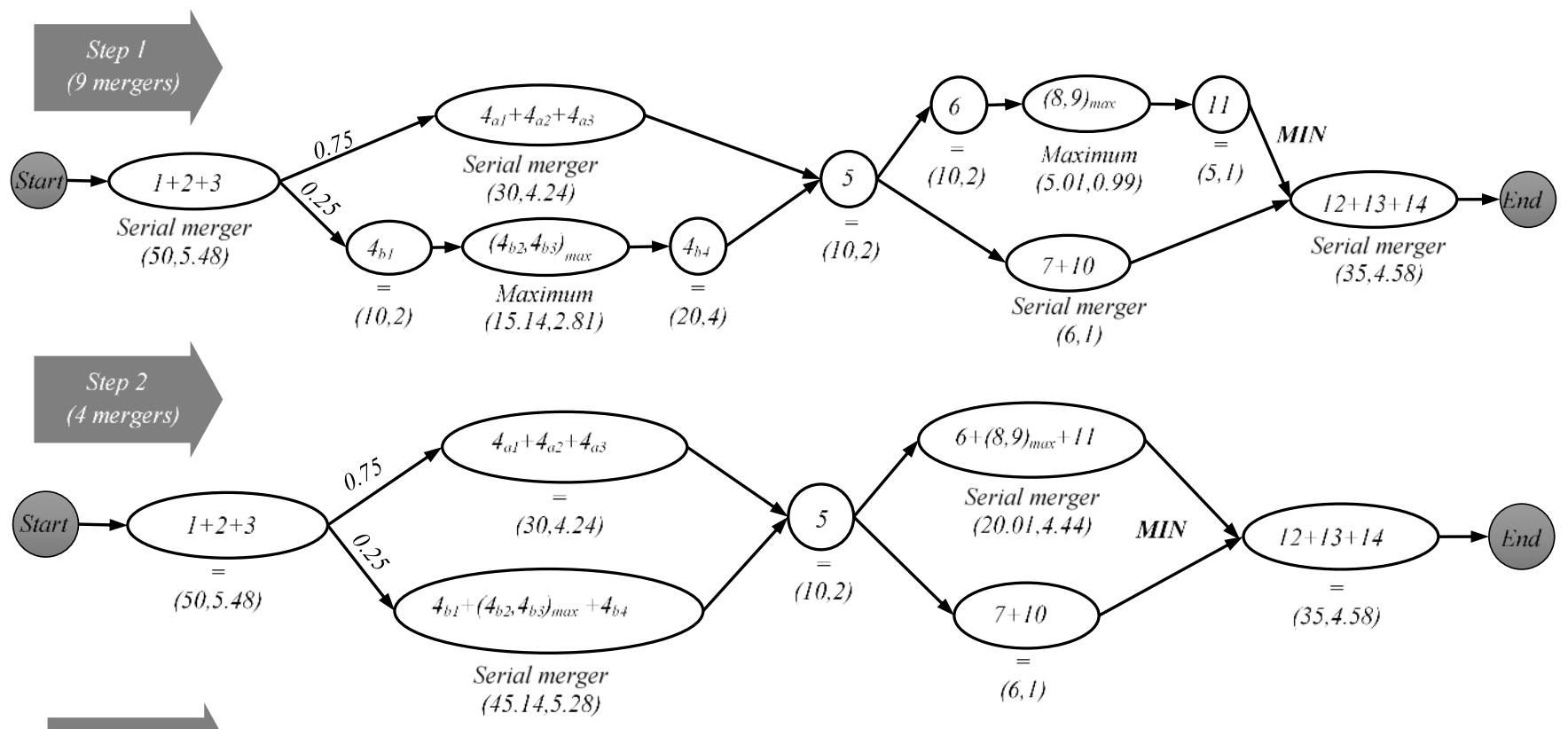

Step 3

(1 merger)
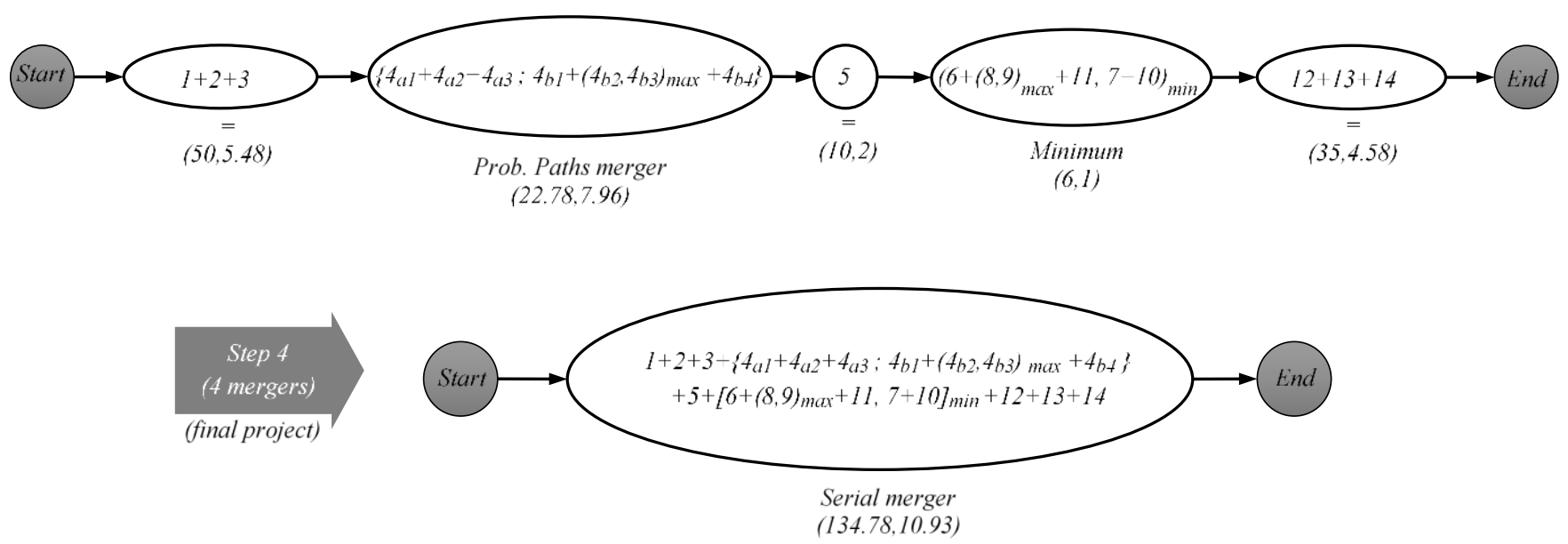


\section{Results}

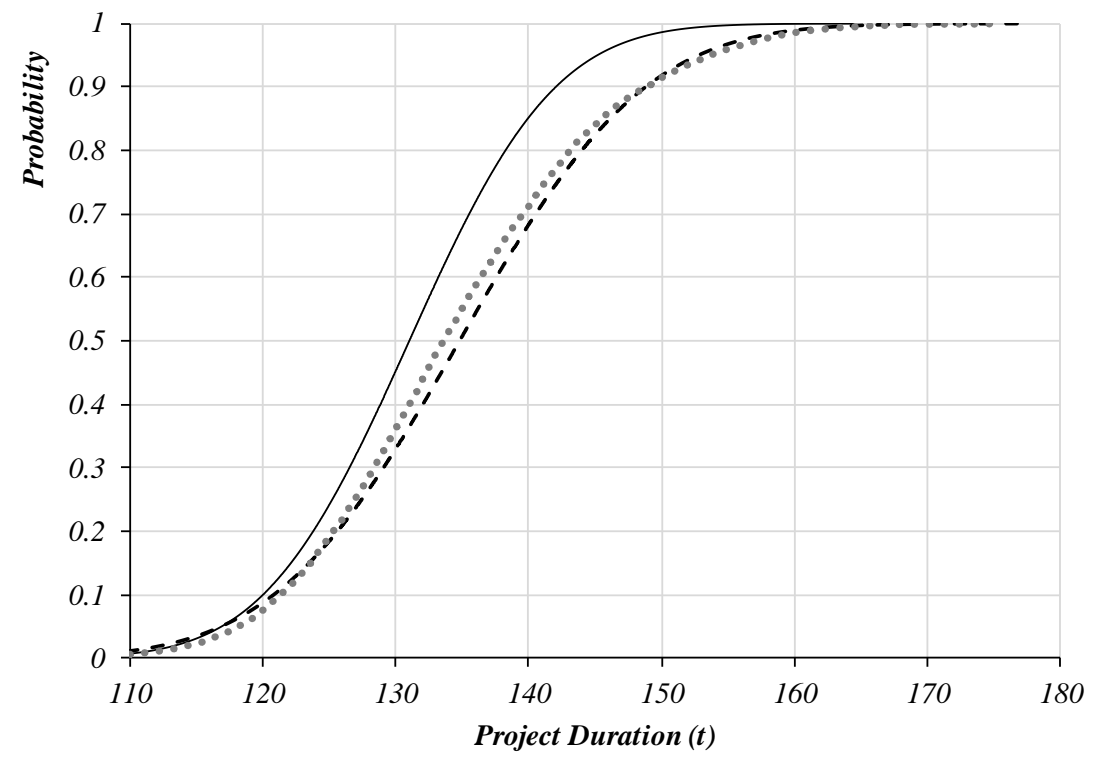

-PERT $\quad--$ M-PERT $\quad \cdots$ Actual distrib 